\title{
Planetary population synthesis
}

\author{
Christoph Mordasini
}

\begin{abstract}
In stellar astrophysics, the technique of population synthesis has been successfully used for several decades. For planets, it is in contrast still a young method which only became important in recent years because of the rapid increase of the number of known extrasolar planets, and the associated growth of statistical observational constraints. With planetary population synthesis, the theory of planet formation and evolution can be put to the test against these constraints. In this review of planetary population synthesis, we first briefly list key observational constraints. Then, the work flow in the method and its two main components are presented, namely global end-to-end models that predict planetary system properties directly from protoplanetary disk properties and probability distributions for these initial conditions. An overview of various population synthesis models in the literature is given. The sub-models for the physical processes considered in global models are described: the evolution of the protoplanetary disk, the planets' accretion of solids and gas, orbital migration, and N-body interactions among concurrently growing protoplanets. Next, typical population synthesis results are illustrated in the form of new syntheses obtained with the latest generation of the Bern model. Planetary formation tracks, the distribution of planets in the mass-distance and radius-distance plane, the planetary mass function, and the distributions of planetary radii, semimajor axes, and luminosities are shown, linked to underlying physical processes, and compared with their observational counterparts. We finish by highlighting the most important predictions made by population synthesis models and discuss the lessons learned from these predictions - both those later observationally confirmed and those rejected.
\end{abstract}

Christoph Mordasini

Physikalisches Institut, University of Bern, Gesellschaftsstrasse 6 CH 3012 Bern, Switzerland. email: christoph.mordasini@space.unibe.ch 


\section{Confronting theory and observation}

Since the discovery of the first extrasolar planet around a solar-like star by Mayor \& Queloz (1995), it has become clear from observations that the population of extrasolar planets is characterized by extreme diversity. This diversity in terms of planetary masses, orbital distances, system architectures, internal compositions etc. was not anticipated by earlier theoretical models of planet formation (e.g., Boss 1995) that were based on just one planetary system, our own solar system, indicating important shortcomings in the theory.

Since 1995, the number of known extrasolar planets has increased rapidly, reaching now several thousand (Schneider et al. 2011; Wright et al. 2011). This allows one to study the extrasolar planets as a statistical population instead of single objects only, even though the study of a benchmark individual planetary systems (including the solar system) continues to be key to understand planet formation as well. This planetary population is characterized by a number of statistical distribution (e.g., of the mass or eccentricity), dependencies on host star properties (like the stellar metallicity), and correlations between these quantities. These statistical constraints provide a rich data set with which the theoretical predictions of population synthesis models can be confronted.

The basic idea behind the planetary population synthesis method is that the observed diversity of extrasolar planets is due to a diversity in the initial conditions, the protoplanetary disks (e.g., Andrews et al. 2010). While it is typically difficult to observe the process of planet formation directly (except for a handful special cases, e.g., Sallum et al. 2015), in a numerical model the link between disk and planetary system properties can be established with so-called global model. This class of models directly predicts the final (potentially observable) properties of synthetic extrasolar planets based on the properties of their parent synthetic protoplanetary disk. For this, such global end-to-end models build on simplified results of many different detailed models for individual physical processes of planet formation, like accretion and migration. In global models, these individual processes are linked together, which is a source of considerable complexity, even for relatively simple sub-models. Thanks to this approach, the population-wide, statistical consequences of an individual physical description (like orbital migration, e.g., Masset \& Casoli 2010) become clear and can be statistically compared with the observed population (e.g., the semimajor axis distribution or the frequency of mean motion resonances). This means that first, theoretical models of a specific process can be put to the observational test which is otherwise often difficult as we can only observe the combined effect of all acting processes, and second that the full wealth of observational data (the entire statistical information coming from different observational techniques like radial velocities, transits, direct imaging, microlensing, ...) can be used to constrain theoretical planet formation models. This also avoids that models are constructed that can only describe specific types of systems, but fail for many others, as illustrated for the case of the solar system mentioned above.

As global models in population syntheses are in the end nothing else than coupled agglomerates of other specialized models, it is clear that the predictions of 
population syntheses directly reflect the state of the field of planet formation theory as a whole, which is exactly their purpose. This means that as our understanding of planet formation changes, so do the population synthesis models.

In planet formation, fundamental physical processes governing planet formation are currently still uncertain. An important example for this are the processes that drive accretion in protoplanetary disk (classical MRI-driven viscous accretion, MHD winds, e.g., Bai 2016), which has via different disk structures strong consequences for planet formation (Ogihara et al. 2015). Another important example is the relative importance of solid building blocks of various sizes ranging from $\mathrm{cm}$-sized pebbles to classical $100 \mathrm{~km}$-sized planetesimals (Ormel 2017). It could appear that given such large uncertainties, currently no meaningful global models can therefore be constructed - but actually, the argument must be turned around: it is with population syntheses that these different theories can be put to the observational test to identify which ones lead to synthetic populations that agree or disagree with observations, and to improve in this way the understanding of how planets form, which is the final goal.

This chapter is organized as follows: we first discuss the most important observational constraints, then describe the method of population synthesis including a short overview of the input physics currently considered in global models. We then turn to the discussion of the most important results including the comparison with observations. We conclude the chapter with the discussion of tests of specific submodels and predictions for future instruments and surveys.

For further information on the method, the reader may consider the reviews of Benz et al. (2014) and Mordasini et al. (2015). Two other relevant publications for the (initial) development of the method are Ida \& Lin (2004a) and Mordasini et al. (2009a).

\section{Statistical observational constraints}

The number and type of observational statistical constraints available for comparisons is in principle very large and multifaceted (for reviews, see Udry \& Santos 2007; Winn \& Fabrycky 2015). However, there are a number of key constraints the comparison to which population syntheses have traditionally focussed on.

These key constraints are usually the results of large observational surveys, both from the ground and space. Important surveys and publications analyzing them are, e.g., the HARPS high precision radial velocity survey (Mayor et al. 2011), the Keck \& Lick radial velocity survey (Howard et al. 2010), the CoRoT (Moutou et al. 2013) and Kepler transit surveys (Coughlin et al. 2016), the various direct imaging (Bowler 2016), or the microlensing surveys (Cassan et al. 2012). The high importance of surveys stems from the fact that they have a well known observational bias. This makes it possible to correct for it and to infer the underlying actual distributions that are predicted by the theoretical models. 
All these different techniques put constraints on different aspects of the global models. Especially when they are combined, they are highly constraining even for global models that often have a significant number of free parameters as the combined data carries so much constraining information.

The constraints can be grouped into three classes: the frequency of different planet types, the distribution functions of planetary properties, and correlations with stellar properties. We next give a short overview of these observational constraints.

\section{Frequencies of planet types}

- The frequency of hot Jupiters around solar-like stars is about 0.5-1\% (Howard et al. 2010; Mayor et al. 2011).

- The frequency of giant planets within 5-10 AU is $10-20 \%$ for FGK stars (Cumming et al. 2008; Mayor et al. 2011). The giant planets have a multiplicity rate of about 50\% (Bryan et al. 2016).

- There is a high frequency (20-50\%) of close-in (fractions of an AU) low-mass (a few Earth masses) respectively small $\left(R \lesssim 4 R_{\oplus}\right)$ super-Earth and sub-Neptunian planets from high-precision radial velocity (Mayor et al. 2011) and the Kepler survey (Fressin et al. 2013; Petigura et al. 2013). These planets are often found in tightly packed multiple systems. Planetary systems clearly different from the solar system are thus very frequent.

- There is a low frequency on the $1 \%$ level of detectable (i.e., sufficiently luminous) massive giant planets at distances of tens to hundreds of AU. This means that the frequency of giant planets must somewhere drop with orbital distance by about a factor ten. The occurrence rate is likely positively correlated with the stellar mass Bowler (2016).

- There is a high frequency of cold, roughly Neptunian-mass planets around M dwarfs as found by microlensing surveys (Cassan et al. 2012).

- There is a very high total fraction of stars with detectable planets of $\sim 75 \%$ as indicated by high-precision radial velocity searches with a $\sim 1 \mathrm{~m} / \mathrm{s}$ precision (Mayor et al. 2011). At least in the solar neighborhood, stars with planets are thus the rule.

\section{Distributions of planetary properties}

- One of the most important diagrams is the observed two-dimensional distribution of planets in the mass-distance (or radius-distance) plane, revealing a number of pile-ups and deserts (see Fig. 1). For the comparison with the synthetic populations, it is of paramount importance to keep in mind that the observed diagram gives a highly distorted impression of the actual population because of the detection biases of the different techniques. Hot Jupiters, for example, appear to be 


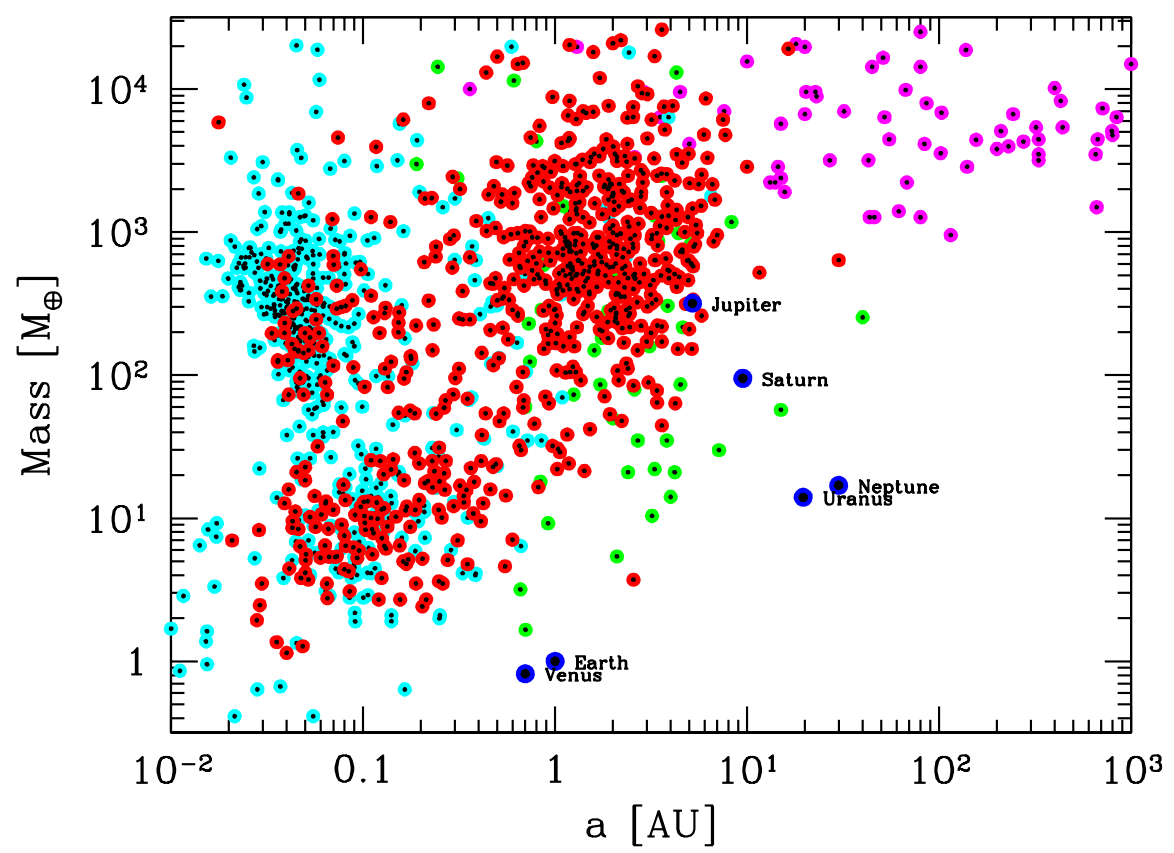

Fig. 1: Mass-distance diagram of confirmed planets in "The Extrasolar Planets Encyclopedia" (Schneider et al. 2011) as of 2017. Red, cyan, magenta, and green points indicate planets detected by the radial velocity, transits, direct imaging, and microlensing technique, respectively. The planets of the solar system are also shown for comparison.

frequent in this plot. But the plot still illustrates the enormous diversity in the outcome of the planet formation process. At the same time, it also indicates that there is some structure.

- The mass function is approximately flat in log space in the giant planet regime (Marcy et al. 2005) for masses between $30 M_{\oplus}$ and about $4 M_{4}$ (where $1 M_{4}$ is the mass of Jupiter). At even higher masses, there is a drop in frequency (Santos et al. 2017). The upper end of the planetary mass function is poorly known, but might lie around $30 M_{4}$ (Sahlmann et al. 2011). Towards the lower masses, at around $30 M_{\oplus}$, there is a break in the mass function and a strong increase of the frequency towards smaller masses (Mayor et al. 2011). The mass function below a few Earth masses is currently unknown.

- The semimajor axis distribution of giant planets consists of a local maximum at a period around 4 days caused by the hot Jupiters, a less populated region further out (the period valley) and finally an upturn at around 1 AU (Udry et al. 2003). The frequency seems to be decreasing beyond 3-10 AU (Bryan et al. 2016).

- The eccentricity distribution is, in contrast to the solar system with its very low eccentricities, broad, including some planets with eccentricities that exceed 0.9. 
The upper part of the distribution follows approximately a Rayleigh distribution, as expected from gravitational planet-planet interactions (Jurić \& Tremaine 2008), indicating together with several other points that in some systems strong dynamical interactions occurred (see the discussion in Winn \& Fabrycky 2015). A significant fraction of orbits are however also consistent with being circular. Eccentricities of lower mass planets $\left(\lesssim 30 M_{\oplus}\right)$ are usually restricted to lower values $\leq 0.5$ (Mayor et al. 2011).

- The radius distribution of confirmed (Kepler) planets has a local maximum at around 1 Jovian radius as expected from the theoretical mass-radius relation (Mordasini et al. 2012b), followed by a distribution that is approximately flat in $\log (R)$ at intermediate radii of 4-10 $R_{\oplus}$. Below this radius, there is strong increase in frequency (Fressin et al. 2013; Petigura et al. 2013). At about $1.7 R_{\oplus}$, there is a local minimum in the radius histogram (Fulton et al. 2017) separating super-Earths from sub-Neptunes. This could be due to atmospheric escape of primordial H/He envelopes (Owen \& Wu 2017; Jin \& Mordasini 2018).

\section{Correlations with stellar properties}

- The best known correlation of planetary and stellar properties is the increase of the frequency of giant planets with host star metallicity (Gonzalez 1997; Santos et al. 2004; Fischer \& Valenti 2005). In the super-solar metallicity domain, the frequency of giant planet increases approximately by a factor ten when going from $[\mathrm{Fe} / \mathrm{H}]=0$ to $[\mathrm{Fe} / \mathrm{H}]=0.5$. This is often taken as indication that core accretion is the dominant mode of giant planet formation (Ida \& Lin 2004b; Mordasini et al. 2012a). The frequency of low-mass planets is in contrast independent of metallicity (Mayor et al. 2011).

- The frequency of giant planets is lower for lower mass stars and around $2 \%$ for M-dwarfs (Bonfils et al. 2013). For stellar masses higher than $1 M_{\odot}$, the frequency first increases to reach a maximum at around $2 M_{\odot}$, followed by a rapid drop for $M_{*} \gtrsim 2.7 M_{\odot}$ (Reffert et al. 2015).

- Statistical correlations with stellar age are not yet well explored, but a number of detections of close-in planets around T-Tauri and young PMS stars have occurred (Mann et al. 2016; David et al. 2016; Donati et al. 2016; Yu et al. 2017). They show that close-in massive planets already exist after a few Myr, likely indicating orbital migration via planet-disk interactions. Hot Jupiters might be more frequent around T Tauri stars than main sequence stars (Yu et al. 2017). At large orbital distances, direct imaging also probes young planets with ages of a few 10 Myr. The PLATO survey will put statistical constraints on the temporal evolution of the population of transiting planets, adding a new temporal dimension to the constraints. 


\section{Population synthesis method}

In this section, we review the general workflow in the method, the past development of population syntheses models, the physical processes considered in global formation and evolution models, and finally the probability distributions of the initial conditions.

\section{Workflow of the population synthesis method}

The general workflow of the planetary population synthesis method is shown in Fig. 2. There are three main elements: first, and most importantly, the global model that predicts planetary properties directly based on disk properties. Second, the Monte Carlo distributions for the initial conditions of the global models that are derived from disk observations, from reconstructions of the disk properties in an equivalent way as done for the minimum mass solar nebula, or from theoretical arguments. Third, tools to apply the observational detection bias and to conduct the statistical comparison with the observed population. In general, this comparison will reveal differences between the theory and observations, which are then tracked back to assumptions about the governing physical processes as implemented in the model as well as the setting of model parameters. In case that the synthetic population matches the observations at least regarding a certain aspect, the synthetic population can also be used to make predictions about aspects that cannot yet be observed, including the expected yield of future surveys.

\section{Overview of population synthesis models in the literature}

In other fields of astrophysics, (stellar) population synthesis is a well-established technique for several decades (e.g., Bruzual \& Charlot 2003), while for planets, it is still a recent approach. The construction of planetary population synthesis models was triggered by the rapidly increasing number of known extrasolar planets. In this section, we review past and present developments of such models by various groups. Early models were all based on the classical core accretion paradigm where the solids are accreted in the form of planetesimals (Perri \& Cameron 1974; Mizuno 1980; Bodenheimer \& Pollack 1986; Pollack et al. 1996). More recently, models were also based on core accretion with pebbles (Ormel \& Klahr 2010; Johansen \& Lambrechts 2017), and on planet formation via gravitational instability (Kuiper 1951; Cameron 1978; Boss 1997).

1. The Ida $\&$ Lin models. The pioneering population synthesis calculation of Ida $\&$ Lin (2004a) contained for the first time all the basic elements of population synthesis shown in Fig. 2, namely a purpose-built - and therefore fast - global 


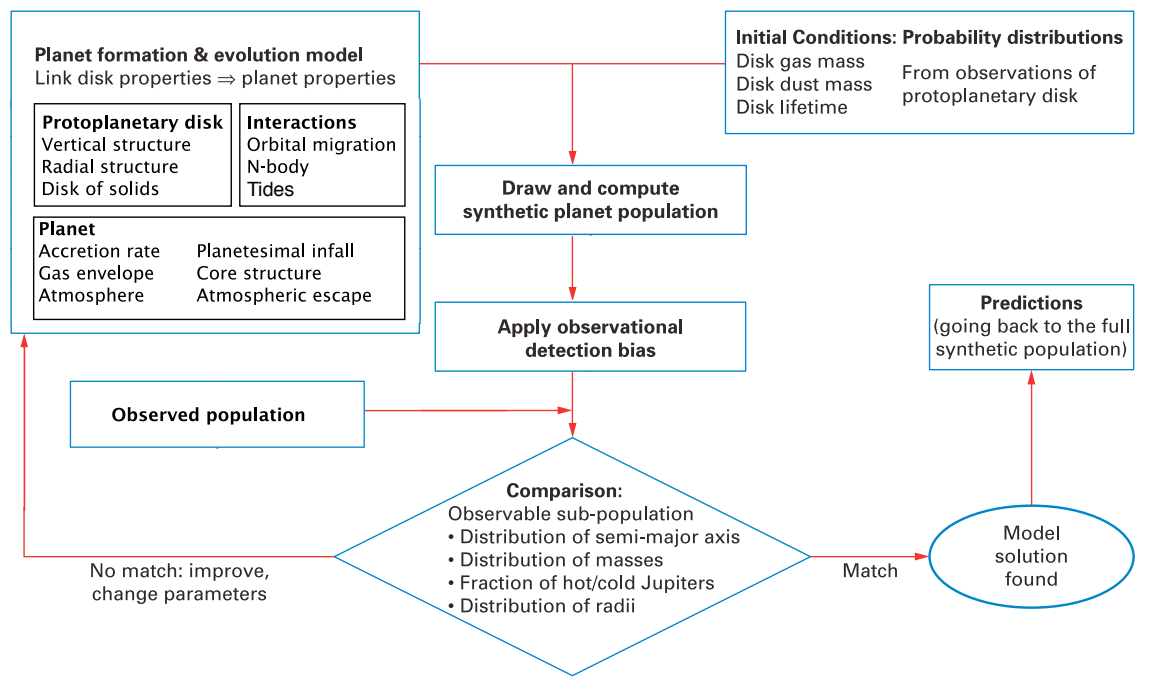

Fig. 2: Elements and work flow of a planetary population synthesis framework (updated from Mordasini et al. 2015).

planet formation model based on the core accretion paradigm, and a variation of the initial conditions in a Monte Carlo way. The effects of planetesimal accretion, parameterized gas accretion, and Type II orbital migration in simple power-law disks were considered. As most first-generation population synthesis models, the one-embryo-per-disk approximation was used. Later works added Type I migration (Ida \& Lin 2008a), a density enhancement due to a dead zone at the iceline (Ida \& Lin 2008b), and finally a semi-analytical statistical treatment of the dynamical interactions of several concurrently growing protoplanets (Ida \& Lin 2010; Ida et al. 2013).

2. The Bern Model. Building on the Alibert, Mordasini \& Benz (2004; 2005) model for giant planet formation in the solar system, Mordasini et al. (2009a,b) presented population syntheses that included quantitative statistical comparisons with observations. Compared to the Ida \& Lin models, the Bern model explicitly solves the (partial) differential for the structure and evolution of the protoplanetary disk and the planets' interior structure, rather then using power-law solutions. This has the implication of substantially higher computational costs. Subsequent improvements addressed the structure of the protoplanetary disk (Fouchet et al. 2012), the solid accretion rate (Fortier et al. 2013), and the type I migration description (Dittkrist et al. 2014). The model was extended to include the planets' post-formation thermodynamic evolution (cooling and contraction) over Gyrs timescales (Mordasini et al. 2012c), as well as atmospheric escape (Jin et al. 2014). This makes it possible to predict directly also radii and luminosities instead of masses only. Also these models originally used the one-embryo-per- 
disk approximation. The concurrent formation of multiple protoplanets interacting via an explicit N-body integrator was added in Alibert et al. (2013).

3. The models of Hasegawa \& Pudritz $(2011,2012)$; Hasegawa \& Pudritz (2013) combine a planet formation model based initially on the Ida\& Lin models with power-law disks with inhomogeneities or the analytical disk model of Chambers (2009) and Cridland et al. (2016). These models emphasize the importance of "planet traps", i.e., special locations in the disk where orbital migration is slowed down or stopped due to transitions in the disk. These transitions are the edge of the MRI-dead zone, icelines, and the transition from the viscously heated to the irradiation-dominated region in the disk. Later updates (Alessi et al. 2017; Cridland et al. 2017) include models for the dust physics, astrochemistry, and radiative transfer.

4. While not used in population syntheses but in parameter studies, the models of Hellary \& Nelson (2012); Coleman \& Nelson (2014); Coleman \& Nelson (2016) are global models that combine an N-body integrator with a 1D model for the disk's structure and evolution and the planets' orbital migration. In contrast to other models, the planetesimals are directly included in the N-body as massless test particles, and not simply represented as a surface density. Early models use fits to the results of Movshovitz et al. (2010) for the planets' gas accretion rate while later models (Coleman et al. 2017) calculate it by solving 1D structure equations. Similar global models were also presented by Thommes et al. (2008).

5. Based on the global model of Bitsch et al. (2015b), Ndugu et al. (2018) presented population syntheses based on the core accretion paradigm where the cores grow by the accretion of pebbles instead of planetesimals. Ndugu et al. (2018) focussed on the effect of the stellar cluster environment. The gas disk structure is obtained from 2D simulations including viscous heating and stellar irradiation assuming a radially constant mass flux (Bitsch et al. 2015a). The planets' gas accretion rate is given by analytical results of Piso \& Youdin (2014). The cores grow by the accretion of mm-cm sized drifting pebbles (Lambrechts \& Johansen 2012; Lambrechts \& Johansen 2014). The model uses the one-embryo-per-disk approach, such that $\mathrm{N}$-body interactions are neglected, while type I and II migration are included.

6. An increasing number of population synthesis calculations are also based on variants of the gravitational instability model for giant planet formation (e.g., Forgan \& Rice 2013; Nayakshin \& Fletcher 2015; Müller et al. 2018). Similar to the core accretion models, these global models couple simple semi-analytic submodels of disc evolution, disk fragmentation, initial embryo mass, gas accretion and loss (for example by tidal downsizing, Nayakshin 2010), orbital migration, grain growth, formation of solid cores by sedimentation, and recently, the N-body interaction of several fragments (Forgan et al. 2018).

In the remainder of the article we concentrate on the population synthesis models based on the core accretion paradigm. 


\section{Global models: simplified but linked}

The core accretion paradigm states that giant planets form in a two-step process. First a so-called critical core is built (with a mass of about $10 M_{\oplus}$ ), which then triggers the accretion of the gaseous envelope. This happens in evolving disks of gas and solids in which also other protoplanets grow, leading to dynamical interactions. The gas disk and the protoplanets exchange angular moment, so that orbital migration occurs. All these processes occur on similar timescales, meaning that they need to be considered in a self-consistently coupled fashion. A global planet formation model must thus consider this minimal set of physical processes (Benz et al. 2014):

1. The structure and evolution of the protoplanetary gas disk

2. The structure and evolution of the disk of solids (dust, pebbles, planetesimals)

3. The accretion of solids leading to the growth of the planetary solid core

4. The accretion of $\mathrm{H} / \mathrm{He}$ leading to the growth of the planetary gaseous envelope

5. Orbital migration resulting from the exchange of angular momentum

6. N-body interaction among (proto)planets

Additional sub-models may describe the internal structure of the core and envelope, the structure of the planetary atmosphere, the interaction of infalling planetesimals and pebbles with the protoplanets' envelope, the evolution of the star, or the loss of the gaseous envelope during the evolutionary phase, for example via atmospheric escape (e.g., Jin et al. 2014).

\section{Low-dimensional approximation}

For a statistical approach like population synthesis where hundreds of planetary systems must be simulated over timescales of many millions of years during the formation epoch, and even for billions of years during the evolution phase, it is currently not possible to use detailed multi-dimensional hydrodynamic simulations possibly even including radiative transfer and magnetic fields because of computational time limitations.

Instead, the sub-models are either parameterized based on the results of detailed models or solve differential equations describing low-dimensional approximations like 1D spherically symmetric hydrostatic planet interior equations or 1D axisymmetric protoplanetary disk evolution equations. A key challenge of population synthesis is thus to "distill" the insights from 2D or 3D detailed models of one specific process (like orbital migration or pebble accretion) into simpler computationally efficient approximations, that however still correctly capture the essence of the governing physical mechanism. Exploring which approximations are possible without losing the essence is an ongoing challenge for population synthesis models.

On the other hand, the fact that the different processes are considered in a selfconsistent coupled fashion over a long timescale is a strong aspect of global models, 
as it captures the non-linear interactions of the different processes as they are occurring also in nature. This coupling is a source of considerable complexity of the models, even for relatively simple individual sub-models.

We next briefly discuss some of these sub-models. More detailed descriptions can be found in Benz et al. (2014) and Mordasini et al. (2017).

\section{Disk models}

The disk model describes the evolution of the surface density of gas and solids. It also gives the gas temperature, pressure, and vertical scale height. These quantities and their radial derivatives enter into the other sub-models in multiple ways, making the disk model a key component of a global model. The gas disk properties for example determine as outer boundary conditions the gas accretion rate of protoplanets, enter into the migration rates, control the aerodynamic behavior of small particles, or the damping of the random velocities of the planetesimals.

The simplest way of setting up a parameterized (gas) disk model is a power law approach inspired by the minimum mass solar nebula MMSN (Weidenschilling 1977; Hayashi 1981), as used in the original models of Ida \& Lin (2004a). In the MMSN approach, the present-day positions, masses, and compositions of the solar system planets are used to reconstruct the radial distribution of matter in the solar nebula, assuming in situ growth. In such models, the (initial) surface density of gas $\Sigma$ as a function of distance from the star $r$ is given as

$$
\Sigma(r)=\Sigma_{0}\left(\frac{r}{1 \mathrm{AU}}\right)^{-3 / 2} .
$$

In a population synthesis, the normalization constant $\Sigma_{0}$ is varied as a Monte Carlo variable to represent disk of different masses (see Sect. Probability distribution of disk initial conditions) with $\Sigma_{0} \approx 2400 \mathrm{~g} / \mathrm{cm}^{2}$ corresponding for example to the surface density in the MMSN. In such simple models, the temperature $T$ is also given as a power law. (Ida \& Lin 2004a) for example assumed an optically thin disk, and a main sequence-scaling of the stellar luminosity as $L \propto M_{\star}^{4}$ with stellar mass, so that

$$
T(r)=280 \mathrm{~K}\left(\frac{r}{1 \mathrm{AU}}\right)^{-1 / 2}\left(\frac{M_{\star}}{M_{\odot}}\right) .
$$

This however neglects (a) that disks are optically thick (both radially and vertically) with opacity transitions at condensation fronts which can act as migration traps, (b) the effect of viscous heating, and (c) it does not include any temporal evolution, including the fact that stars are not yet on the main sequence during the presence of the gas disk. A certain improvement over such simple MMSN-like models are analytical disk models that take these effects into account, as for example the Chambers (2009) disk model that distinguishes between an inner viscously heated part and an outer irradiation-dominated part. 
A more complex, but still 1D approach is to solve the classical viscous evolution equation (Lüst 1952; Lynden-Bell \& Pringle 1974) for the surface density of the gas as a function of time $t$ and distance from the star $r$

$$
\frac{\partial \Sigma}{\partial t}=\frac{1}{r} \frac{\partial}{\partial r}\left[3 r^{1 / 2} \frac{\partial}{\partial r}\left(r^{1 / 2} v \Sigma\right)\right]-\dot{\Sigma}_{\text {phot }}(r)-\dot{\Sigma}_{\text {planet }}(r) .
$$

with a viscosity $v$ that is written in the $\alpha$-parameterization as $v=\alpha c_{\mathrm{S}} H$ with $c_{\mathrm{S}}$ the sound speed and $H$ the vertical scale height (Shakura \& Sunyaev 1973). Besides the viscous evolution term, the effects of mass loss by photoevaporation (e.g., Alexander et al. 2014) represented by $\dot{\Sigma}_{\text {phot }}(r)$ and of gas accretion by the planets giving raise to the $\dot{\Sigma}_{\text {planet }}(r)$ term are also to be included.

As an initial condition for this equation, the gas surface density is assumed to consist of a decrease close to the star due to the stellar magnetospheric cavity, a power-law in the main part, and an exponential decrease outside of a characteristic radius, as found in the analytical solution to the viscous accretion disk problem of Lynden-Bell \& Pringle (1974). The initial gas surface density is then

$$
\Sigma_{\mathrm{g}}(t=0, r)=\Sigma_{0}\left(\frac{r}{1 \mathrm{AU}}\right)^{p_{\mathrm{g}}} \exp \left[-\left(\frac{r}{R_{\text {out }}}\right)^{2+p_{\mathrm{g}}}\right]\left(1-\sqrt{\frac{r}{R_{\text {in }}}}\right) .
$$

In this equation, $R_{\text {out }}$ is the "characteristic" (outer) disk radius, $R_{\text {in }}$ the inner radius, and $p_{\mathrm{g}}$ the power law exponent. Observations indicate $p_{\mathrm{g}} \approx-1$ (Andrews et al. 2010). The four parameters in this equation may be treated as Monte Carlo random variables in a population synthesis.

Under the assumption that dust is converted early in the disk's evolution everywhere with full efficiency into planetesimals, the initial surface density of planetesimals $\Sigma_{\mathrm{p}}$ would be given as (Mordasini et al. 2009a)

$$
\Sigma_{\mathrm{p}}(t=0, r)=f_{\text {dg }} \eta_{\text {ice }} \Sigma_{\mathrm{g}}(t=0, r)
$$

where $f_{\text {dg }}$ is the dust-to-gas ratio ( $\approx$ the heavy element mass fraction $Z$ ), which is about 0.0149 in the Sun (Lodders 2003). It is another Monte Carlo variable, representing the different metallicities of stars (see Fig. 4). Finally, $\eta_{\text {ice }}$ reflects the reduction of the solid surface density at iceline(s).

However, observations (e.g., Panić et al. 2009) and theoretical results (e.g., Birnstiel et al. 2012) indicate that a significant radial redistribution of solids in the form of pebbles occurs. This may lead to more concentrated and steeper distributions of the solids (Kornet et al. 2001; Birnstiel \& Andrews 2014) than predicted by Eq 5. In pebble-based models, the pebble surface density is calculated from the radial flux of pebbles, which is in turn controlled by the production rate of pebbles from dust at the pebble production line (Bitsch et al. 2015b).

The temporal evolution of the gas disk is found by solving the aforementioned equation describing a viscous accretion disk including photoevaporation. In parameterized models like in Ida \& Lin (2004a), one uses instead an equation of the form 
Planetary population synthesis

$$
\dot{\Sigma}_{\mathrm{g}}(r)=-\frac{\Sigma_{\mathrm{g}}(r)}{\tau_{\text {disk }}}+\dot{\Sigma}_{\text {phot }}
$$

The first term on the right hand side leads to an exponential self-similar decay, while the second mimics the effects of photoevaporation. The characteristic disk timescale $\tau_{\text {disk }}$ can again be treated as a Monte Carlo variable (Sect. Probability distribution of disk initial conditions).

The surface density of solids decreases within the planet's feeding zone according to the amount of mass that the planet accretes, assuming that the surface density is uniform within the feeding zone (Thommes et al. 2003), i.e.,

$$
\dot{\Sigma}_{\mathrm{p}}=-\frac{\left(3 M_{*}\right)^{1 / 3}}{6 \pi a_{\mathrm{p}}^{2} B_{L} M_{\mathrm{p}}^{1 / 3}} \dot{M}_{\mathrm{c}}
$$

where $B_{L}$ is the width of the feeding zone in Hill spheres, $M_{\mathrm{p}}$ the planet's mass, $a_{\mathrm{p}}$ its semimajor axis, and $\dot{M}_{\mathrm{c}}$ the planet's planetesimals accretion rate.

\section{Accretion of solids}

In planetesimal based models, the growth of the solid core with mass $M_{\mathrm{c}}$ is assumed to occur in the classical picture via the accretion of small background planetesimals. For this, a version of the Safronov (Safronov 1969) equation which gives the core accretion rate $\dot{M}_{c}$ is used:

$$
\dot{M}_{\mathrm{c}}=\Omega \Sigma_{\mathrm{p}} R_{\text {capture }}^{2} F_{\mathrm{G}}
$$

where $\Omega$ is the Keplerian frequency, $\Sigma_{\mathrm{p}}$ the mean surface density of planetesimals in the planet's feeding zone, $R_{\text {capture }}$ the capture radius which is in general larger than the core radius because of gas drag (Podolak et al. 1988; Mordasini et al. 2006), and $F_{\mathrm{G}}$ is the gravitational focussing factor (Nakazawa et al. 1989; Greenzweig \& Lissauer 1992). It depends among other quantities on the random velocities of the planetesimals $v_{\text {pls }}$ and would be given in the (idealized) two-body case as $1+\left(v_{\mathrm{esc}} / v_{\mathrm{pls}}\right)^{2}$, where $v_{\text {esc }}$ is the escape velocity from the protoplanet (Safronov 1969). The random velocities of smaller planetesimals are more strongly damped by nebular gas drag leading to a higher focussing factor in Eq. 8. Furthermore, the drag-enhanced capture radii of the protoplanets in Eq. 8 is increased as well for smaller bodies, approaching very large radii for small particles as exemplified by pebble accretion.

An insight into the dependencies of the core accretion rate on parameters can be obtained by considering the core accretion timescale $\tau_{\mathrm{c}}$ in

$$
\dot{M}_{\mathrm{c}}=\frac{M_{\mathrm{c}}}{\tau_{\mathrm{c}}} .
$$

Based on the work of Kokubo \& Ida (2002), Ida \& Lin (2004a) derive an approximate expression for the accretion timescale in the oligarchic growth regime. In the oligarchic regime, the random velocities of the planetesimals is raised by viscous stirring by the protoplanet, while it is damped by gas drag during the presence of 
the gas disk (Ida \& Makino 1993). This regimes occurs after an initial runaway planetesimal accretion phase as soon as the protoplanets have grown to a size of 100-1000 km depending on orbital distance (Ormel et al. 2010).

The accretion timescale in this regime is

$$
\begin{aligned}
\tau_{\mathrm{c}}=1.2 \times 10^{5} \mathrm{yr}\left(\frac{\Sigma_{\mathrm{p}}}{10 \mathrm{~g} \mathrm{~cm}^{-2}}\right)^{-1}\left(\frac{a_{\mathrm{p}}}{1 \mathrm{AU}}\right)^{1 / 2}\left(\frac{M_{c}}{M_{\oplus}}\right)^{1 / 3}\left(\frac{M_{\star}}{M_{\odot}}\right)^{-1 / 6} \times \\
{\left[\left(\frac{\Sigma_{\mathrm{g}}}{2400 \mathrm{~g} \mathrm{~cm}^{-2}}\right)^{-1 / 5}\left(\frac{a_{\mathrm{p}}}{1 \mathrm{AU}}\right)^{1 / 20}\left(\frac{m}{10^{18} \mathrm{~g}}\right)^{1 / 15}\right]^{2} . }
\end{aligned}
$$

In this equation $\Sigma_{\mathrm{g}}$ is the gas surface density at the planet's position at $a_{\mathrm{p}}$, and $m$ is the mass of a planetesimal.

We see from Eq. 10 that the growth is faster at smaller distances as the collisional growth scales with the orbital frequency leading to growth wave propagating outward. The timescale also increases as $M_{\mathrm{c}}^{1 / 3}$ as typical for the oligarchic regime, and decreases inversely proportional to $\Sigma_{\mathrm{p}}$. This faster growth, and the fact that cores can also become more massive at higher $\Sigma_{\mathrm{p}}$ (e.g., Kokubo \& Ida 2012) explains why the core accretion theory predicts a higher number of giant planets at higher $[\mathrm{Fe} / \mathrm{H}]($ see Sect. Correlations with disk properties).

In pebble based models (see, e.g., Ormel 2017), the accretion of pebble sets in once the protoplanets have reached a size where the encounter with the pebbles transitions from the ballistic to the settling regime. In the former, gas-drag effects are not relevant, whereas in the settling regime the encounter time is sufficiently long to allow the incoming particles to couple aerodynamically to the gas, and to sediment towards the protoplanet during the encounter. This leads to an efficient, gas-drag-aided accretion. This transition occurs at one AU when the protoplanets have reached a size of several hundred $\mathrm{km}$, increasing with orbital distance (Visser \& Ormel 2016).

\section{Accretion of gas}

Two approaches are used in global models to calculate a protoplanet's gas accretion rate. The first more complex approach taken for example in the Bern model is to calculate the interior structure of the (proto)planets (Alibert et al. 2005; Mordasini et al. 2012c). The planets' interior is modeled by integrating numerically the $1 \mathrm{D}$ spherically symmetric structure equations which are the mass conservation, hydrostatic, energy conservation, and energy transport equations (Bodenheimer \& Pollack 1986):

$$
\begin{array}{lll}
\frac{\partial m}{\partial r}=4 \pi r^{2} \rho & \frac{\partial P}{\partial r}=-\frac{G m}{r^{2}} \rho \\
\frac{\partial l}{\partial r}=4 \pi r^{2} \rho\left(\varepsilon-P \frac{\partial V}{\partial t}-\frac{\partial u}{\partial t}\right) & \frac{\partial T}{\partial r}=\frac{T}{P} \frac{\partial P}{\partial r} \nabla(T, P)
\end{array}
$$


where $r$ is the radius measured from the planet's center, $m$ the enclosed mass, $P$ the pressure, $\rho$ the density, and $G$ the gravitational constant. The gradient $\nabla$ depends on the process by which the energy is transported (radiation or convection). The energy equation is the only equation that is time $t$ dependent and controls the temporal evolution. In this equation, $V=1 / \rho$ is the specific volume, $u$ the specific internal energy, $\varepsilon$ an energy source like impact or radiogenic heating, and $l$ is the intrinsic luminosity.

These structure equations are solved with different outer boundary conditions depending on the phase a protoplanet is in (Bodenheimer et al. 2000; Mordasini et al. 2012c). In the first so-called attached (or nebular) phase, the envelope transitions smoothly into the background nebula, such that the outer pressure and temperature are approximately equal to the local disk pressure and temperature. In this phase, the outer radius is given as the minimum of a fraction of the Hills sphere and the Bondi radius, and can thus be found if the planet's mass is known. Radiative cooling allows the gas in the protoplanetary envelope to contract. This (formally) results in an empty shell between the planet's outer edge of the envelope and the surrounding nebula. This is filled in by new nebular gas, allowing the envelope mass to increase. This means that during the attached phase, the gas accretion rate is regulated by the envelope's cooling (Kelvin-Helmholtz) timescale as found by solving the structure equations.

When the core reaches a mass of about $10 M_{\oplus}$, the contraction of the envelope becomes so rapid that the protoplanetary disk can no longer supply gas at a rate sufficient to keep the envelope and disk in contact (runaway gas accretion leading to giant planet formation). The planet's outer radius now detaches from the nebula and contracts rapidly, but still quasi-statically (Bodenheimer \& Pollack 1986) to a radius that is much smaller than the Hills sphere (about $1.5-5 R_{4}$ depending on the entropy, Mordasini et al. 2012c; Mordasini et al. 2017). In this second so-called detached (or transition) phase, the radius is free and found by solving the structure equations, while the gas accretion rate is given by processes in the protoplanetary disk and no longer by the envelope's contraction. This disk-limited rate may be given by the Bondi accretion rate (D'Angelo \& Lubow 2008; Mordasini et al. 2012c)

$$
\dot{M}_{\mathrm{e}, \mathrm{Bondi}} \approx \frac{\sum_{\mathrm{g}}}{H}\left(\frac{R_{\mathrm{H}}}{3}\right)^{3} \Omega
$$

where $\Sigma_{\mathrm{g}}, H, R_{\mathrm{H}}$, and $\Omega$ are the mean gas surface density in the planet's feeding zone, the disk's vertical scale height, the planet's Hill sphere radius, and the orbital frequency at the planet's position. It is also possible to calculate the planet's disklimited gas accretion rate as a fraction $f_{\text {lub }}$ of the local viscous accretion rate in the disk, which is in equilibrium given as

$$
\dot{M}_{\mathrm{e}, \mathrm{visc}}=f_{\text {lub }} 3 \pi v \Sigma_{\mathrm{g}} .
$$

Hydrodynamical simulations (Lubow et al. 1999) indicate that $f_{\text {lub }}$ may be as high as 0.9 meaning that the planet accretes $90 \%$ of the local gas flow through the disk. At higher masses, gap formation starts to reduce the gas accretion rate, leading to a 
reduction which can be fitted as (Veras \& Armitage 2004)

$$
f_{\text {va04 }}=1.668\left(\frac{M_{\mathrm{p}}}{M_{\text {Jup }}}\right)^{1 / 3} \exp \left(-\frac{M_{\mathrm{p}}}{1.5 M_{\text {Jup }}}\right)+0.04 .
$$

The second simpler approach used by other global models (e.g., Ida \& Lin 2004a; Hasegawa \& Pudritz 2012; Ndugu et al. 2018) to calculate the gas accretion rate is based on fits for the KH-timescale. The gas accretion rate due to the contraction of the envelope is approximated as

$$
\dot{M}_{\mathrm{e}, \mathrm{KH}}=\frac{M_{\mathrm{p}}}{\tau_{\mathrm{KH}}}
$$

where the Kelvin-Helmholtz cooling timescale of the envelope is parameterized as (Ikoma et al. 2000)

$$
\tau_{\mathrm{KH}}=10^{p_{\mathrm{KH}}} \operatorname{yr}\left(\frac{M_{\mathrm{p}}}{M_{\oplus}}\right)^{q_{\mathrm{KH}}}\left(\frac{\kappa}{1 \mathrm{~g} \mathrm{~cm}^{-2}}\right)
$$

where $p_{\mathrm{KH}}$ and $q_{\mathrm{KH}}$ are parameters that are obtained by fitting the accretion rate found with internal structure calculations like Bodenheimer \& Pollack (1986); Ikoma et al. (2000); Mordasini et al. (2014). For example, Ida \& Lin (2004a) used $p_{\mathrm{KH}}=9$ and $q_{\mathrm{KH}}=-3$ and neglected the influence of $\kappa$. Mordasini et al. (2014) found $p_{\mathrm{KH}}=10.4, q_{\mathrm{KH}}=-1.5$, and $\kappa=10^{-2} \mathrm{~g} / \mathrm{cm}^{2}$. Once can see from Eq. 17 that the accretion rate is a rapidly increasing function of mass, and that gas accretion becomes important once $\tau_{\mathrm{KH}}$ becomes comparable to, or shorter than, the disk lifetime.

Compared to the method of solving directly the internal structure, the KHmethod is computationally much simpler and more robust. But it cannot take into account how the gas accretion rate depends on (a) the (variable) luminosity of the core because of solid accretion, and (b) the varying outer boundary conditions. This means that it cannot easily recover the spread in associated envelope masses for a fixed core mass visible in Fig. 3. Furthermore it does not yield the planets' internal structure and thus radius and luminosity. But also the solution of the $1 \mathrm{D}$ hydrostatic structure equations is only an approximation as it neglects that protoplanetary envelopes are not closed strictly hydrostatic $1 \mathrm{D}$ systems, but that they can exchange gas with the surrounding disk in a hydrodynamic multi-dimensional manner (Ormel et al. 2015). This can delay gas accretion through the advection of high entropy material (Cimerman et al. 2017). As it is typical for global end-to-end models to rely on low-dimensional approaches (1D or 1+1D) because of computational efficiency, these effects were not considered so far in population syntheses.

Figure 3 shows the envelope mass as a function of core mass at the end of the formation phase in the population around $1 M_{\odot}$ stars presented in Sect. Results. These envelope masses were found by solving the aforementioned 1D internal structure equations assuming a grain opacity in the protoplanetary atmospheres that is 0.003 times as large as the ISM grain opacities (Mordasini et al. 2014), and by limiting the 


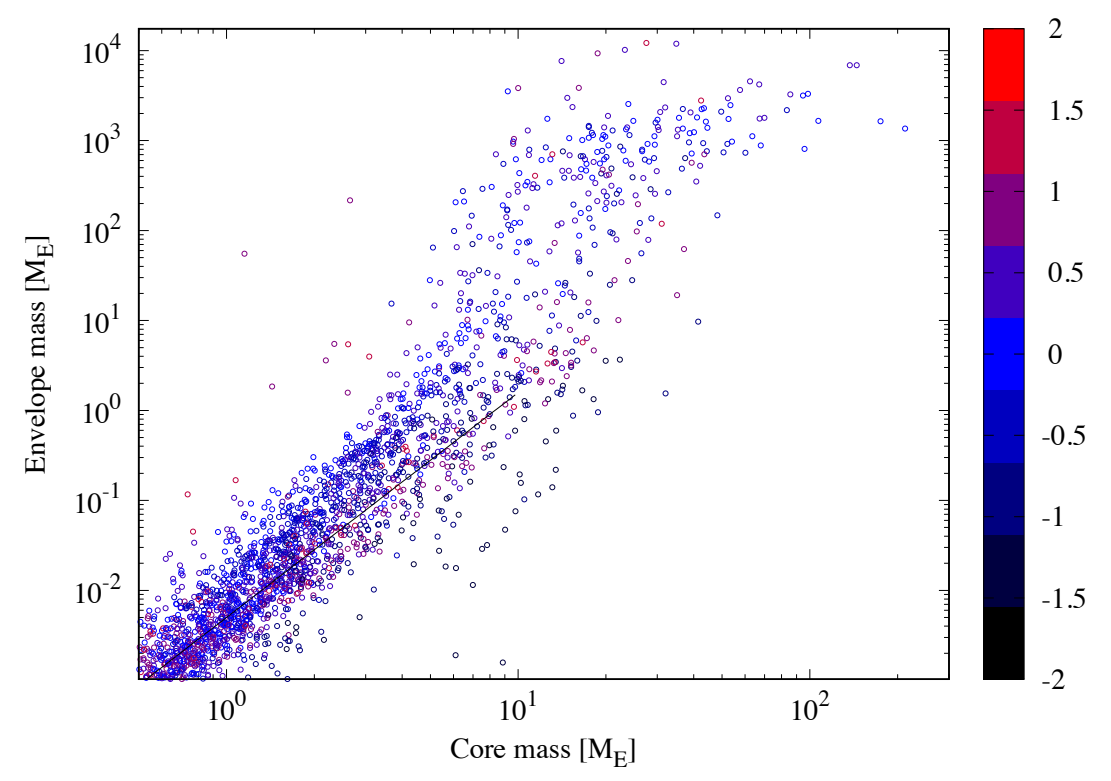

Fig. 3: $\mathrm{H} / \mathrm{He}$ envelope mass as a function of core mass found from solving the internal structure equations for the synthetic population discussed in Sect. Results. The relation is shown at the end of the formation phase when the gaseous disks disperse. The colors show the planets' semimajor axis as $\log (a / \mathrm{AU})$. The black line scales as $M_{\text {core }}^{2.5}$.

gas accretion rate in the disk-limited phase similarly to Eq. 13 (see Mordasini et al. $2012 \mathrm{c}$ for details). At low masses, the envelope mass scales as $M_{\mathrm{c}}^{-q_{\mathrm{KH}}+1}$ (i.e., $M_{\mathrm{c}}^{2.5}$ in the simulation here, indicated by the black line, see also Mordasini et al. 2014). Then, at a core mass of about 5-20 $M_{\oplus}$, gas accretion becomes rapid (runaway accretion), so planets move upwards nearly vertically to higher $M_{\mathrm{e}}$ and become giant planets.

There are fewer planets in the intermediate mass range between about 10 to 100 $M_{\oplus}$. This is because the timescale to accrete this gas mass in runaway accretion is shorter than the disk lifetime, so that it is unlikely that the disk disappears exactly at an intermediate moment/mass. This is the origin of the so called "planetary desert" (Ida \& Lin 2004a). It is weaker in the population here compared to the original Ida $\&$ Lin (2004a) predictions due to the larger effective $q_{\mathrm{KH}}=-1.5$ instead of -3 as used by Ida \& Lin (2004a), meaning that the gas accretion rate does not increase as rapidly with mass, and due to the limits given by the Bondi rate. Regarding the termination of gas accretion, in this simulation, the disk-limited gas accretion decreases in time just because $\Sigma_{\mathrm{g}}$ in the feeding zone (Eq. 13) decreases because of disk evolution. Gas accretion is thus terminated when the gas disk disappears. 


\section{Orbital migration}

The gravitational interaction of the gaseous disk and the embedded protoplanets results in the exchange of angular momentum (for recent reviews see Kley \& Nelson 2012; Baruteau et al. 2016), which means that the planets change their semimajor axis, i.e., the undergo orbital migration (Goldreich \& Tremaine 1979; Ward 1986; Lin \& Papaloizou 1986a). The angular momentum transfer between disk gas and planets via torques leads in most cases to a loss of angular momentum for the planet which means inward migration. The angular momentum $J$ of a planet of mass $M_{\mathrm{p}}$ orbiting a star of mass $M_{*}$ at a semimajor axis $a_{\mathrm{p}}$, and the migration rate $d a / d t$ given a total torque $\Gamma_{\text {tot }}=d J / d t$ are

$$
J=M_{\mathrm{p}} \sqrt{G M_{*} a_{\mathrm{p}}} \quad \frac{d a}{d t}=2 a_{\mathrm{p}} \frac{\Gamma_{\mathrm{tot}}}{J} .
$$

Other effects like planetesimal-driven migration (e.g., Levison et al. 2010; Ormel et al. 2012) or Kozai migration due to an external perturber (Kozai 1962; Fabrycky $\&$ Tremaine 2007) can also modify the orbits, but were up to now not considered in population synthesis models.

Disk-driven migration occurs in two types, Type I and Type II migration. Type I migration occurs if the planet's Hill sphere radius is smaller than the disk's vertical scale height and if the viscous torques are dominant compared to the gravity torques induced by the planet (Crida et al. 2006), meaning that Type I migration applies to low-mass planets. Various descriptions of Type I migration have been derived in the literature. Early derivations (Tanaka et al. 2002) assumed that the disk behaves (locally) isothermal, and predicted rapid inward migration. Later, more realistic calculations (e.g., Baruteau \& Masset 2008; Casoli \& Masset 2009; Paardekooper et al. 2010; Kley et al. 2009) directly modeled the cooling behavior of the disk gas. They showed that there are several sub-types of Type I migration (locally isothermal, adiabatic, (un)saturated corotation torque) that can be identified by considering an number of timescales (Dittkrist et al. 2014). An important finding is that for nonisothermal Type I migration, in some parts of the disk outward migration can occur. Therefore, there are special locations like condensation fronts where the torque vanishes because of the associated opacity transitions. Such locations can act as traps for migrating planets (Lyra et al. 2010; Hasegawa \& Pudritz 2011; Sándor et al. 2011; Kretke \& Lin 2012), and can serve as locations of efficient planetary growth (e.g., Horn et al. 2012; Hasegawa \& Pudritz 2012).

To calculate the torque causing a planet's migration, one needs among other quantities like the gas surface density the local power law exponent of the disk temperature $p_{\mathrm{T}}$ and of the gas surface density $p_{\Sigma}$, which are yielded by the disk model, underlining its importance. The migration timescale in the isothermal approximation used by Ida \& Lin (2008a) is given as

$$
\tau_{\text {typeI }}=\frac{1}{2.728+1.082 p_{\Sigma}}\left(\frac{c_{\mathrm{s}}}{a_{\mathrm{p}} \Omega}\right)^{2} \frac{M_{*}}{M_{\mathrm{p}}} \frac{M_{*}}{a_{\mathrm{p}}^{2} \Sigma_{\mathrm{g}}} \Omega^{-1}
$$


and the migration rate is then

$$
\dot{a}_{\mathrm{p}}=-\frac{a_{\mathrm{p}}}{\tau_{\text {typeI }}}
$$

which shows that migration speeds up a planet grow more massive. In a more recent analysis, Paardekooper et al. (2010) found that the total torque $\Gamma_{\text {tot }}$ resulting from summing up the contributions from the inner and outer Lindblad torques plus the corotation torque can be expressed in an equation of the form

$$
\Gamma_{\text {tot }}=\frac{1}{\gamma}\left(C_{0}+C_{1} p_{\Sigma}+C_{2} p_{\mathrm{T}}\right) \Gamma_{0}
$$

with

$$
\Gamma_{0}=\left(\frac{q}{h}\right)^{2} \Sigma_{\mathrm{g}} a_{\mathrm{p}}^{4} \Omega^{2}
$$

where $\gamma$ is the ratio of the heat capacities, $h=H / a_{\mathrm{p}}$ the local disk aspect ratio, $q=M_{\mathrm{p}} / M_{*}, \Sigma_{\mathrm{g}}$ the gas surface density at the planet's position, and $\Omega$ its Keplerian frequency. The constants $C_{i}$ depend on the Type I sub-regime. Their numerical values are listed for example in Dittkrist et al. (2014).

Type II migration occurs if the angular momentum injection rate of the planet into the disk is so large that it carves a gap into the gas disk around its location. For global models, several different descriptions of Type II migration were considered in the literature: Ida \& Lin (2004a) consider the angular momentum transfer rate in a viscous accretion disk without planets (the viscous torque or "couple" in the terminology of Lynden-Bell \& Pringle 1974) and assume that planets in the type II migration regime act as relays that transmit angular momentum also at this rate across their gap via tidal torques. Inserting the viscous torque into Eq. 18, the Type II migration rate is given as

$$
\dot{a}_{\mathrm{p}}=3 \operatorname{sign}\left(a_{\mathrm{p}}-R_{\mathrm{m}}\right) \alpha \frac{\sum_{\mathrm{g}, \mathrm{m}} R_{\mathrm{m}}^{2}}{M_{\mathrm{p}}} \frac{\Omega_{\mathrm{m}}}{\Omega}\left(\frac{H_{\mathrm{m}}}{a_{\mathrm{p}}}\right)^{2} \Omega_{\mathrm{m}}
$$

where quantities with the subscript $m$ are evaluated at the radius of maximum viscous couple (or velocity reversal, i.e., where the disk changes from accreting to decreting, see Lynden-Bell \& Pringle 1974). The position of $R_{\mathrm{m}}$ can either be estimated as in Ida \& Lin (2004a)

$$
R_{\mathrm{m}}=10 \mathrm{AU} \exp \left(\frac{2 t}{5 \tau_{\text {disk }}}\right) .
$$

or results automatically from solving the evolutionary equation for the gas surface density (Eq. 3).

The Type II migration description of Alibert et al. (2005) assumes that a planet follows the motion of the gas except for the case that the planet is massive compared to the local disk mass, when the planet is assumed to slow down because of its inertia (Alexander \& Armitage 2009). The migration rate is thus given as 


$$
\dot{a}_{\mathrm{p}}=u_{\mathrm{r}} \min \left(1, \frac{2 \Sigma_{\mathrm{g}} a_{\mathrm{p}}^{2}}{M_{\mathrm{p}}}\right)
$$

where $u_{\mathrm{r}}$ is the local radial velocity of the accreting gas which is in equilibrium given as $3 v /\left(2 a_{\mathrm{p}}\right)$. A more realistic yet computationally still feasible approach for a population synthesis (i.e., a 1D approach) is to employ the impuls approximation (Lin \& Papaloizou 1986b) to estimate the Type II migration, as for example done in Coleman \& Nelson (2014).

Given recent results (e.g., Duffell et al. 2014; Dürmann \& Kley 2015) questioning the classical conception that planets in Type II migration simply follow the viscous evolution of the disk, but that their migration rate is entirely given by the torques, make it likely that migration models will undergo significant modifications in the future. The same is true for Type I migration, where new effects like an additional "heating" torque resulting from the protoplanet's accretional luminosity counteracts inward migration (Benítez-Llambay et al. 2015), or a "dynamic" corotation torque (Paardekooper 2014; Pierens 2015) that results from the fact that the relative motion of gas and a migrating planet can lead to a feedback (usually, torques are measured for planets at fixed positions). This can lead to outward migration as well.

\section{N-body interactions}

The concurrent formation of several protoplanets in a protoplanetary disk affects the growth history of the protoplanets in multiple ways: the protoplanets compete for the accretion of gas and solids, increase the velocity dispersion of the planetesimals potentially reducing the solid accretion rate of neighboring protoplanets, alter the surface density of planetesimals and for giant planets of the gas, and reduce the radial flux of pebbles. The gravitational interaction between the protoplanetes leads in the case of insufficient damping by the gas disk to the excitation of the eccentricities, resulting in the alteration of the orbits, collisions, and ejections. Orbital migration is affected as well, since the planets can capture into mean motion resonances and migrate together as resonant convoys. For specific parameters, this can even invert the direction of migration (Masset \& Snellgrove 2001) and lead to the outward migration of two giant planets, as invoked for the "grand tack" scenario in the solar system (Walsh et al. 2011).

As discussed above, all early population synthesis models used the one-embryoper-disk approach, which was one of the most important limitation of the first generation of the models, in particular for low-mass planets as they usually occur in multiple systems, often in compact configurations (e.g., Mayor et al. 2011). This means that they likely influenced each other during formation.

This limitation was addressed in Ida \& Lin (2010); Ida et al. (2013) and Alibert et al. (2013). In the Bern model an explicit N-body integrator was added "on top" of the existing sub-models that calculates the N-body interactions and collisions of the concurrently forming protoplanets. In order to keep the computational time sufficiently low for population syntheses, about 20-50 low-mass embryos (0.01-0.1 
$\left.M_{\oplus}\right)$ are put into each disk. They interact via the usual Newtonian N-body forces written in the heliocentric system,

$$
\ddot{\mathbf{r}}_{i}=-G\left(M_{*}+m_{i}\right) \frac{\mathbf{r}_{\mathbf{i}}}{r_{i}^{3}}-G \sum_{j=1, j \neq i}^{n} m_{j}\left\{\frac{\mathbf{r}_{\mathbf{i}}-\mathbf{r}_{\mathbf{j}}}{\left|\mathbf{r}_{\mathbf{i}}-\mathbf{r}_{\mathbf{j}}\right|^{3}}+\frac{\mathbf{r}_{\mathbf{j}}}{r_{j}^{3}}\right\}
$$

with $i=1,2,3 \ldots N$ the planet index, $\mathbf{r}_{\mathbf{i}}$ and $m_{i}$ the heliocentric position and mass of planet $i$, and $M_{*}$ the mass of the central star. The consequences of the gravitational interaction with the gas disk (orbital migration and damping of eccentricities and inclinations) are entered as additional forces into the integrator (Cresswell \& Nelson 2008).

A different approach was taken by Ida \& Lin (2010) and Ida et al. (2013), who developed a new semi-analytical approach to describe the gravitational interactions of several protoplanets in a statistical way based on orbit crossing timescales, including the effect of resonant capture for migrating planets. The advantage of this approach is a computational cost that is orders of magnitude lower than the direct $\mathrm{N}$-body integration, while still yielding distributions of the eccentricities and semimajor axes of interacting planets that agree well with the direct N-body simulations.

\section{Probability distribution of disk initial conditions}

The second central ingredient for a population synthesis calculation are sets of initial conditions (see Fig. 2). These sets of initial conditions are drawn in a Monte Carlo way from probability distributions. These probability distributions represent the varying properties of protoplanetary disks and are derived as closely as possible from results of disk observations, or, if the quantities are not observable, from theoretical arguments. Typically, there are at least four Monte Carlo variables employed (Ida \& Lin 2004a; Mordasini et al. 2009a):

1. The metallicity and dust-to-gas ratio It is usually assumed that the bulk metallicity is identical in the star and its protoplanetary disk. Then, the disk metallicity $[\mathrm{M} / \mathrm{H}]$ can be modeled as a normal distribution as observed spectroscopically in the photosphere of solar-like stars in the solar neighborhood, with $\mu=-0.02$ and $\sigma=0.22$ (Santos et al. 2005). The $[\mathrm{M} / \mathrm{H}]$ is converted into a disk dust-to-gas ratio (Eq. 5) via $f_{\mathrm{dg}}=f_{\mathrm{dg}, \odot} 10^{[\mathrm{M} / \mathrm{H}]}$, with a solar $f_{\mathrm{dg}, \odot}$ of about 0.01 to 0.02 (Lodders 2003). Together with the initial disk gas mass and the locations of icelines, $f_{\mathrm{dg}}$ sets the amount of solids (dust, pebbles, planetesimals) available in the disk for planet formation.

2. The initial disk gas mass The concept of an "initial" disk mass is of course questionable as it results from the dynamical collapse of a molecular cloud core (Shu 1977; Hueso \& Guillot 2005), but it could be associated with the disk's mass at the moment when the main infall phase has ended, and no self-gravitational instabilities occur any more. Stability arguments (Shu et al. 1990), the inferred 

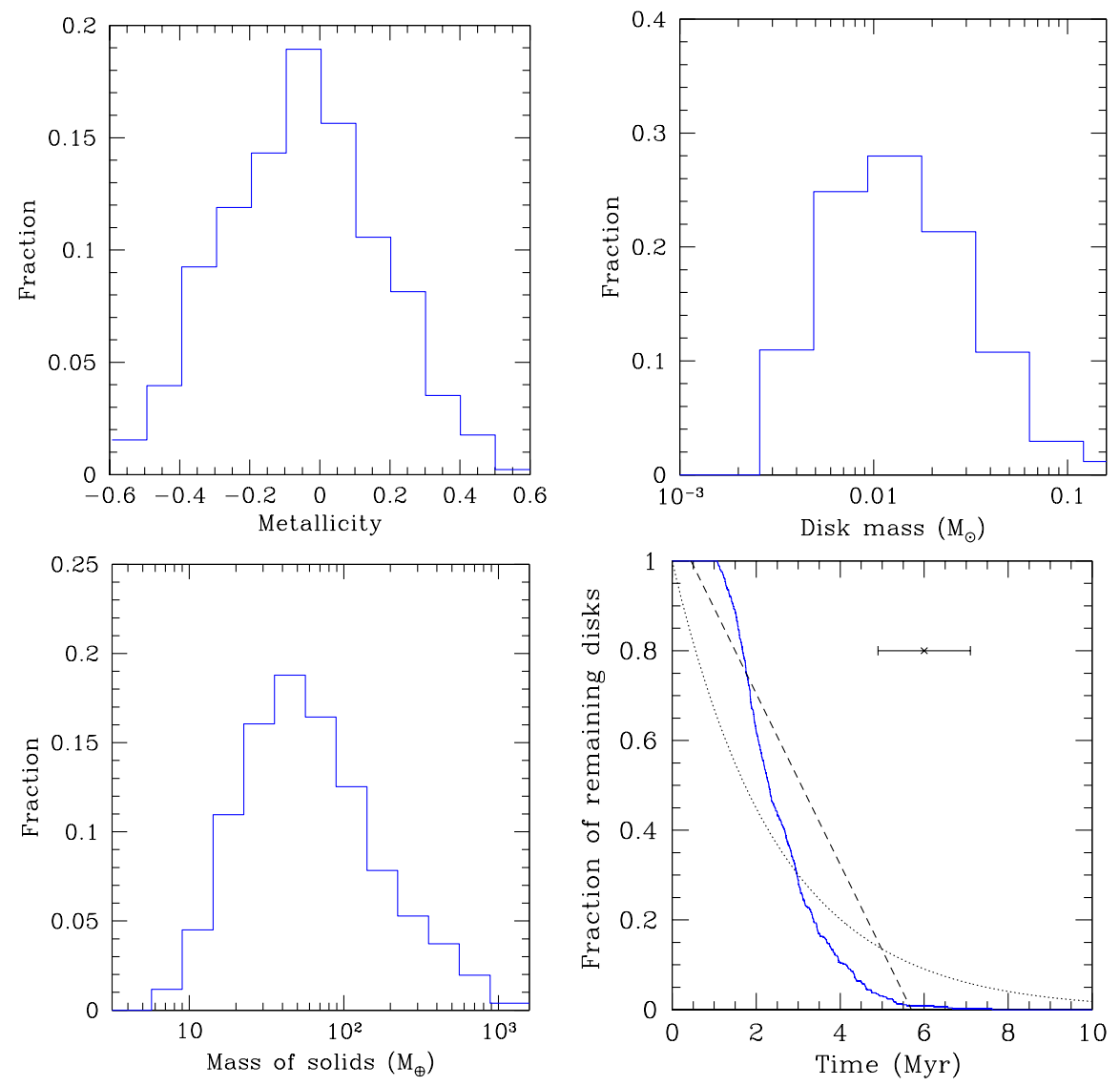

Fig. 4: Distributions of initial conditions for disk around $1 M_{\odot}$ stars. Top left: Metallicity. Top right: initial disk gas mass. Bottom left: initial content of planetesimals. Bottom right: lifetime of synthetic disk (blue). The black solid and dotted lines show observationally determined lifetimes by Haisch et al. (2001) and Mamajek (2009), respectively. The horizontal bars shows the typical observational age uncertainty.

mass of the MMSN (Weidenschilling 1977; Hayashi 1981), and observations of protoplanetary disk (Andrews et al. 2010; Manara et al. 2016) point towards disk masses of about 0.1 to $10 \%$ of the star's mass. The disk masses seem to be distributed roughly log-normally with a mean around $1 \%$ of the star's mass (Mordasini et al. 2009a), but one should note that this distribution is poorly known.

3. The disk lifetime The observations of IR and UV excesses of young stars indicate that the fraction of stars with protoplanetary disks decreases on a timescale of 1-10 Myr, with a mean lifetime of about 3 Myr (Haisch et al. 2001; Mamajek 2009). In a global model, this timescale can either be set directly in Eq. 6, or is used to find a distribution of photoevaporation rates (Eq. 3) that lead together 
with viscous accretion to a distribution of lifetimes of the synthetic disks that agrees with observations.

4. The initial starting positions of the embryos Based on the finding of N-body simulations that oligarchs emerge with relative spacings of a few Hill spheres (Kokubo \& Ida 2000), a distribution of the starting embryos that is uniform in the $\log$ of the semimajor axis is usually used. It is also possible to arrange the embryos such that they "fill" the disk taking into account the asymptotic planetesimal isolation mass (Ida \& Lin 2010). In the trapped evolution models of Hasegawa \& Pudritz (2011); Cridland et al. (2016) embryos rapidly move into traps, so that it is the locus and movement of the traps that effectively gives the formation locations.

Other quantities that may also be treated as Monte Carlo variables are for example the quantities describing the initial radial distribution of the gas and solids in Eq. 4. Other important parameters of the global models like the stellar mass, the planetesimals size, or -for viscous accretion disks- the $\alpha$ viscosity parameter (Shakura \& Sunyaev 1973) are usually kept constant for one synthetic population, but are varied across different populations to understand their statistical impact in parameter studies (e.g., Mordasini et al. 2009b).

Figure 4 shows the distributions of the disk (and stellar) metallicities, initial gas disk masses, the mass of planetesimals initially contained in the disks obtained with $f_{\mathrm{dg}, \odot}=0.02$, and the disk lifetimes. These are the initial conditions for the population synthesis described below in Sect. Results, containing 504 stars with $1 M_{\odot}$. Note that in this model, the disk lifetime is not directly set, but results from the combined action of viscous accretion and an appropriately chosen distribution of photoevaporation rates.

\section{Results}

To illustrate what can be obtained from modern population synthesis calculations, we present in the following sections results from the latest generation of the Bern model. The underlying global formation and evolution model used here is very similar to the model published in Alibert et al. (2013) regarding the N-body interactions of the protoplanets, and to the model published in Mordasini et al. (2012b) regarding the internal structure and long-term thermodynamic evolution (cooling, contraction, envelope evaporation) of the planets. In the new simulations presented here, these two aspects are now combined. This makes it possible to predict not only the orbital elements and masses, but also the radii and luminosities of planets in multiplanet synthetic systems. As differences to the two previously published models, the evolution of the star is now also considered via the Pisa stellar evolution tracks (Dell'Omodarme et al. 2012), and the Mercury N-body integrator (Chambers 1999) is now employed. As in previous models (e.g., Mordasini et al. 2016), the location of the icelines is calculated with the initial disk structure and remains static in time under the assumption that efficient planetesimal formation happens early, and that 
for the 300-m planetesimals, drift is not very important, which should be the case at least in the outer nebula (e.g., Piso et al. 2015). Clearly, this is a strong assumption.

\section{Initial conditions and parameters}

Each system initially contains 20 planetary embryos with a starting mass of $0.1 M_{\oplus}$. These planetary seeds are distributed randomly according to a log-uniform distribution between 0.05 and $40 \mathrm{AU}$. Because of the influence of the initial condition, results concerning synthetic planets that are not clearly more massive than $0.1 M_{\oplus}$ should be regarded with caution. The stellar mass is in all cases $1 M_{\odot}$, and 504 star-disk systems are simulated. The formation phase of the systems was simulated during $10 \mathrm{Myr}$, during which the disks of gas and solid evolve, while the planets accrete mass, migrate, and interact and collide via the N-body integrator. Afterwards, the thermodynamic long-term evolution was calculated for $10 \mathrm{Gyr}$. During this later phase, the planets' mass is constant except for atmospheric escape and no dynamical interactions occur.

The initial gas surface density follows the profile in Eq. 4, while the initial planetesimal follows a steeper profile $\propto r^{-1.5}$ as in the MMSN and a outer exponential radius that is half as large as the one for the gas (in Eq. 4) to account for the inward drift of dust (Kornet et al. 2001; Birnstiel \& Andrews 2014) and the more concentrated distributions resulting from planetesimal formation (Drążkowska et al. 2016). The planetesimal size is 300 meters, and the $\alpha$ viscosity parameter is 0.002 . The grain opacity in the protoplanetary atmospheres during formation is reduced to 0.003 the ISM grain opacity (Mordasini et al. 2014). During evolution, atmospheric opacities of a condensate-free solar-composition gas are assumed (Freedman et al. 2014).

\section{Formation tracks}

Before discussing the statistical results, we present simulations obtained with the global model for one specific system, as the phenomena found in one system often help to understand the statistical results.

Figure 5 illustrates the effect of planetary growth, N-body interaction, and orbital migration (Type I and II) for a system taken from the population described above. The effects of general inward migration, resonant capture, collisions, eccentricity excitation by planet-planet interaction, as well as eccentricity damping because of the gas disk can be seen. Starting from 20 embryos that are interacting via the Nbody integrator, the system in the end contains 2 giant planets, a hot Neptunian planet, and 1 inner and 2 outer low-mass planets.

Orbital migration reduces the orbital distance for several planets by up to a factor 10. The existence of Type I migration traps at opacity transitions (Sect. Orbital mi- 


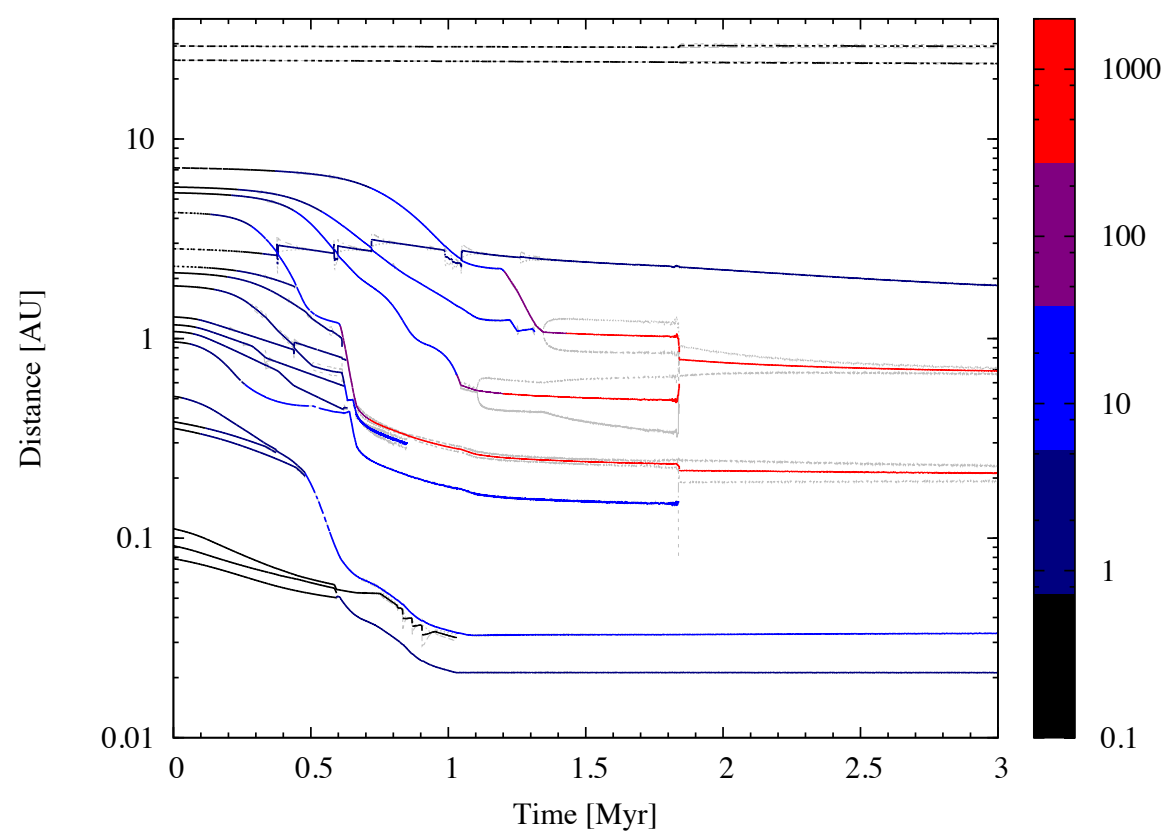

Fig. 5: Inward migration, growth, and dynamical interaction in a synthetic system containing initially 20 planetary embryos of $0.1 M_{\oplus}$ The system is taken from the population synthesis described at the beginning of this section. The tracks of the planets in the time versus orbital distance plane are shown. The black-blue-red lines show the planets' semimajor axes, with the color code representing the planets' mass in Earth masses. The grey lines show the apocenter and pericenter. Lines end when the corresponding protoplanet was either accreted by another body, or ejected because of dynamical interactions.

gration), the slowing down of Type II migration because of the giant planets' inertia (Eq. 25), and the finite disk lifetime still prevent the planets from falling into the star.

Figure 6 shows growth tracks in the distance-mass plane in the same synthetic system as in Fig. 5. Several effects can be seen: at the beginning, the accretion timescale of solids is much shorter than the migration timescale, leading to nearly vertically rising tracks. With increasing mass, the solid accretion timescale (Eq. 10) becomes longer, while the migration timescale becomes shorter (Eq. 19), so the the planets start to migrate inwards at nearly constant mass once they have grown to about 5-10 $M_{\oplus}$. Some very low-mass planets are also captured into MMRs and pushed inwards by more massive protoplanets.

Three protoplanets grow so massive that they trigger runaway gas accretion occurring when $M_{\text {core }} \approx M_{\text {env }} \approx 10 M_{\oplus}$ as visible from the color code. During gas runaway, the growth tracks are again nearly vertical. Finally, the N-body interaction 


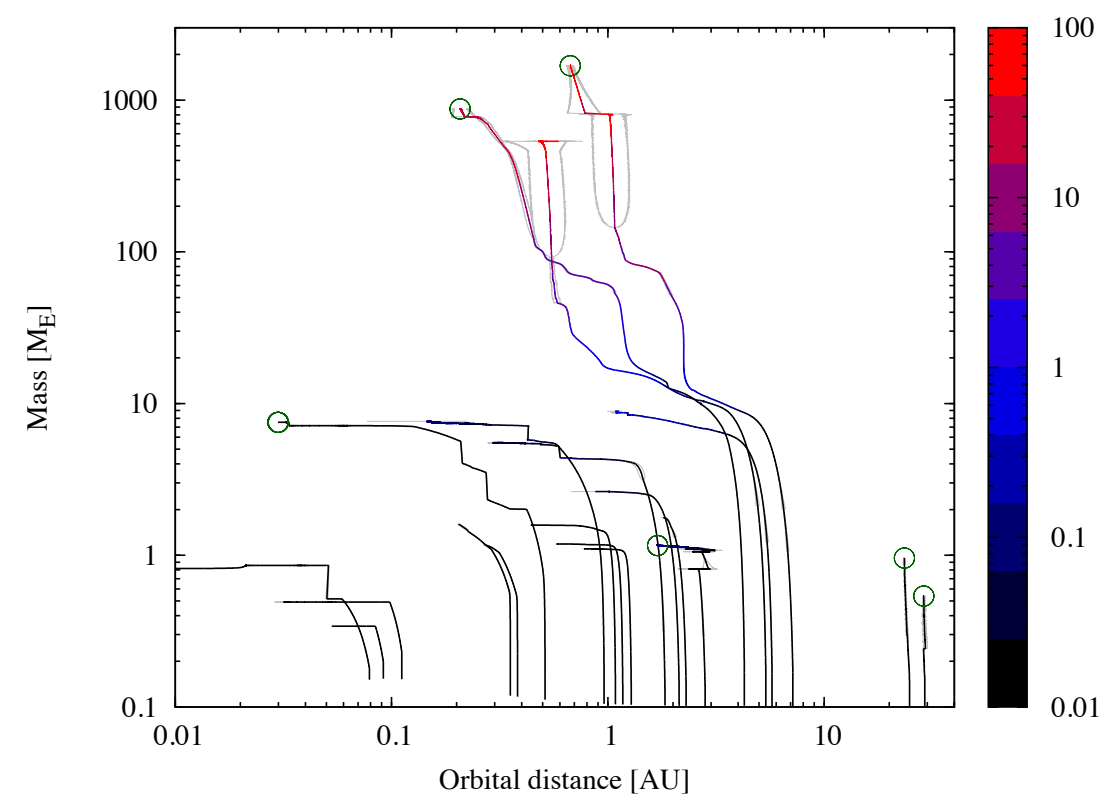

Fig. 6: Growth tracks in the distance-mass plane in the same synthetic system as in Fig. 5. The colored lines show the semimajor axis, color coding the ratio of the $\mathrm{H} / \mathrm{He}$ envelope mass relative to the to core mass $M_{\text {env }} / M_{\text {core }}$. Gray lines show the apocenter and pericenter distances. Open circles show the final position of the remaining planets.

between the three giant planets increases their eccentricities until their orbits overlap, as visible from the gray lines in Fig. 5 and 6. At about $1.9 \mathrm{Myr}$, this leads to the ejection of one giant planet that was located at about 0.5 AU between the two surviving ones.

\section{Diversity of planetary system architectures}

The specific outcome in the simulation shown in Fig. 5 and 6 depends obviously on the initial conditions, and only shows one possible realization. To illustrate the architecture of planetary systems resulting from the global model and the distributions of initial conditions described in Sect. Probability distribution of disk initial conditions, we show in Fig. 7 the final mass-distance diagram of 23 selected synthetic planetary systems. The systems are ordered from bottom left to top right according to increasing metallicity $[\mathrm{M} / \mathrm{H}]$. Note that the systems were selected by hand to display the diversity of architectures, and give the incorrect impression that systems with giant planets are common. This is not the case: only about $18 \%$ of all systems 
have a giant planet (see Sect. Comparison with observations: planet frequencies). To first order, the systems can be split in three classes:

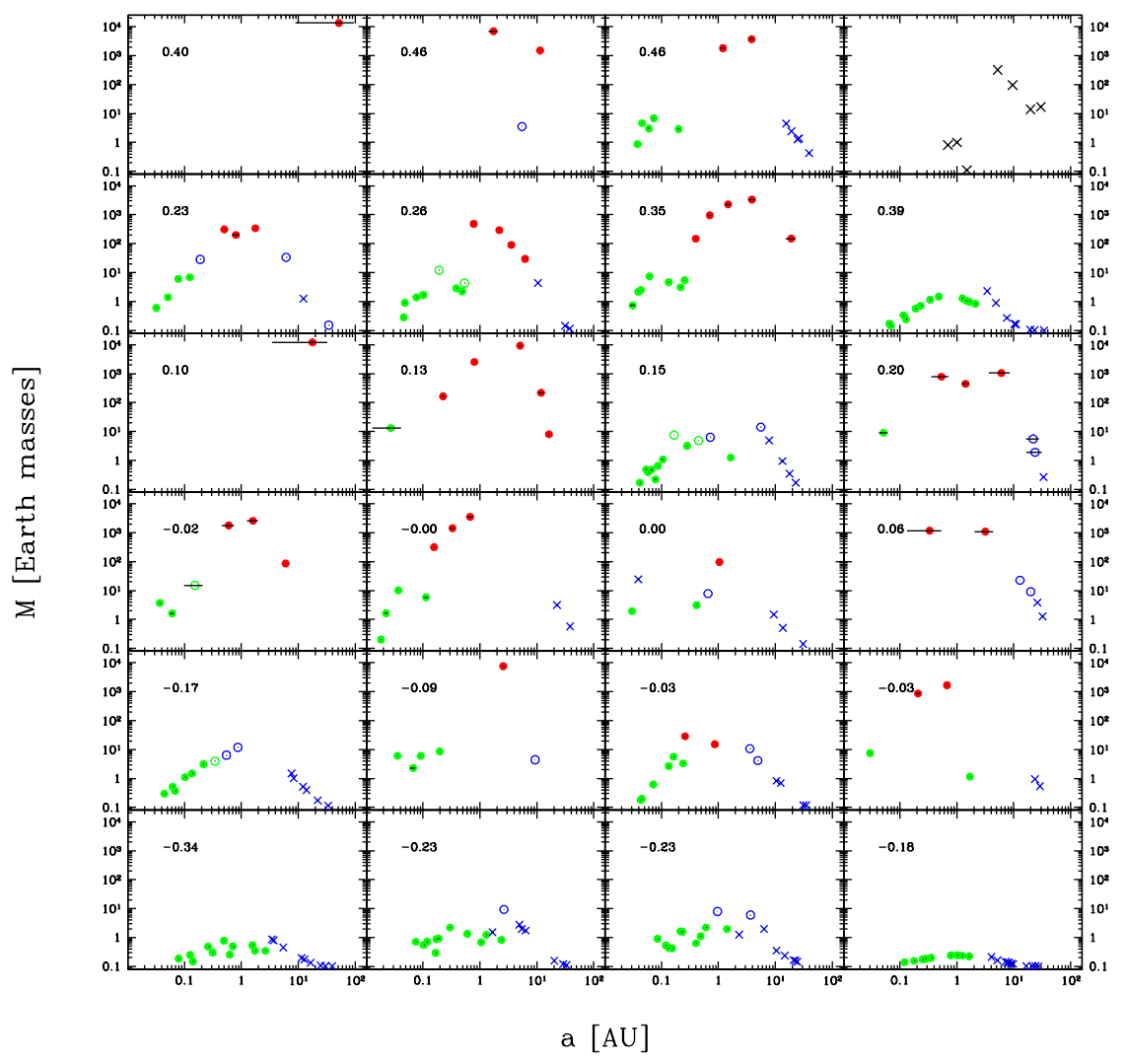

Fig. 7: 23 selected synthetic planetary systems in the mass-semimajor axis plane for $1 M_{\odot}$ stars. The system is taken from the population synthesis described at the beginning of this section. Systems are ordered according to increasing $[\mathrm{M} / \mathrm{H}]$, the number in each panel. Red points are giant planets with $M_{\text {env }} / M_{\text {core }}>1$. Blue symbols are planets that have (partially) accreted volatile material (ices) outside of the iceline(s), while green symbols have only accreted refractory solids. Open green and blue circles have $0.1 \leq M_{\text {env }} / M_{\text {core }} \leq 1$. Filled green points and blue crosses have $M_{\text {env }} / M_{\text {core }} \leq 0.1$. The black horizontal bars go from $a-e$ to $a+e$. The top right panel is the solar system for comparison.

(1) The large majority of the systems are similar to those visible in the bottom left corner: they only contain low-mass planets, with masses of 0.1-10 $M_{\oplus}$. These are systems where the disk properties are such (low surface densities of gas and solids, short disk lifetime) that only little growth occurs during the first 10 Myr. Not much 
orbital migration and dynamical interaction has occurred because of the low planetary masses. Note that further growth on long timescales after $10 \mathrm{Myr}$ is neglected in the model. This could first lead to further accretion, and second it could reduce the number of planets by giant impacts (in these systems the number of final planets is close to the initial number of embryos). The systems have a simple compositional transition from rocky to icy with increasing distance. The more massive planets $\left(\sim 5-10 M_{\oplus}\right)$ have accreted gas envelopes comparable to Uranus and Neptune, otherwise little gas accretion has occurred (and was partially lost after formation by atmospheric escape which is modeled as described in Jin et al. 2014). This type of system is preferentially forming at subsolar $[\mathrm{M} / \mathrm{H}]$, but note that they also exist at high $[\mathrm{M} / \mathrm{H}]$ (for example the $[\mathrm{M} / \mathrm{H}]=0.15$ and 0.39 systems). This makes clear that all four Monte Carlo variables play an important role in determining the outcome of the formation process.

(2) Already much less common are systems with giant planets and low-mass planets. Some contain only rocky low-mass planets inside of the giant planets (the $[\mathrm{M} / \mathrm{H}]=-0.02$ system), some only icy planets outside of them (the $[\mathrm{M} / \mathrm{H}]=0.06$ system). Some are also reminiscent of the solar system and contain both inner terrestrial planets and outer icy planets (see the $[\mathrm{M} / \mathrm{H}]=-0.00$ system). Such a small-largesmall arrangement is the classical outcome for collisional growth from planetesimals with little orbital migration (or redistribution of solids in general): inside, the low availability of solids prevents much growth, while outside it is the long growth timescale that keeps planet masses low. The sweet spot for giant plant growth is a region outside of the water iceline. The architecture of the giant planets varies significantly: the number of giant planets in a system varies from 1 to 5 ; in some systems the giants' mass increases with distance, in others it increases, and in some it is fairly constant; the eccentricities also range from near-zero value in many cases to higher values of about 0.2 .

However, despite the diversity, there is one common characteristic that distinguishes almost all synthetic systems from the solar system: the innermost giant planet is clearly closer-in than Jupiter, namely at about $1 \mathrm{AU}$ or even less. This is an intriguing result. For the solar system, the "grand tack" model (Walsh et al. 2011) suggests that Jupiter (and Saturn) also migrated to about 1.5 AU, to then migrate outward because of the Masset-Snellgroove effect occurring for resonantly coupled giants (Masset \& Snellgrove 2001). The way Type II migration is calculated in the model here from the gas' radial velocity (Eq. 25) does not allow such outward migration. It will be interesting to see whether the inclusion of more realistic migration models (see Sect. Orbital migration) will also lead to more distant synthetic giant planets.

(3) In a small fraction of systems, only one massive giant planet remains at the end. Such systems form in metal-rich and massive disk where several giant planets form in vicinity, leading to violent planet-planet scattering. In Plot 7, in the $[\mathrm{M} / \mathrm{H}]=0.10$ and 0.40 systems such giant planets with masses exceeding $30 \mathrm{M}_{4}$ can be seen with semimajor axes of 20-50 AU and high eccentricities. This gives them pericenter distances of less than $10 \mathrm{AU}$ where the scattering occurred and apocenter distances approaching in one case of about $100 \mathrm{AU}$. They may be detectable by 
direct imaging. In such systems, all other planets were either accreted, sent into the star, or ejected.

The a-M distribution

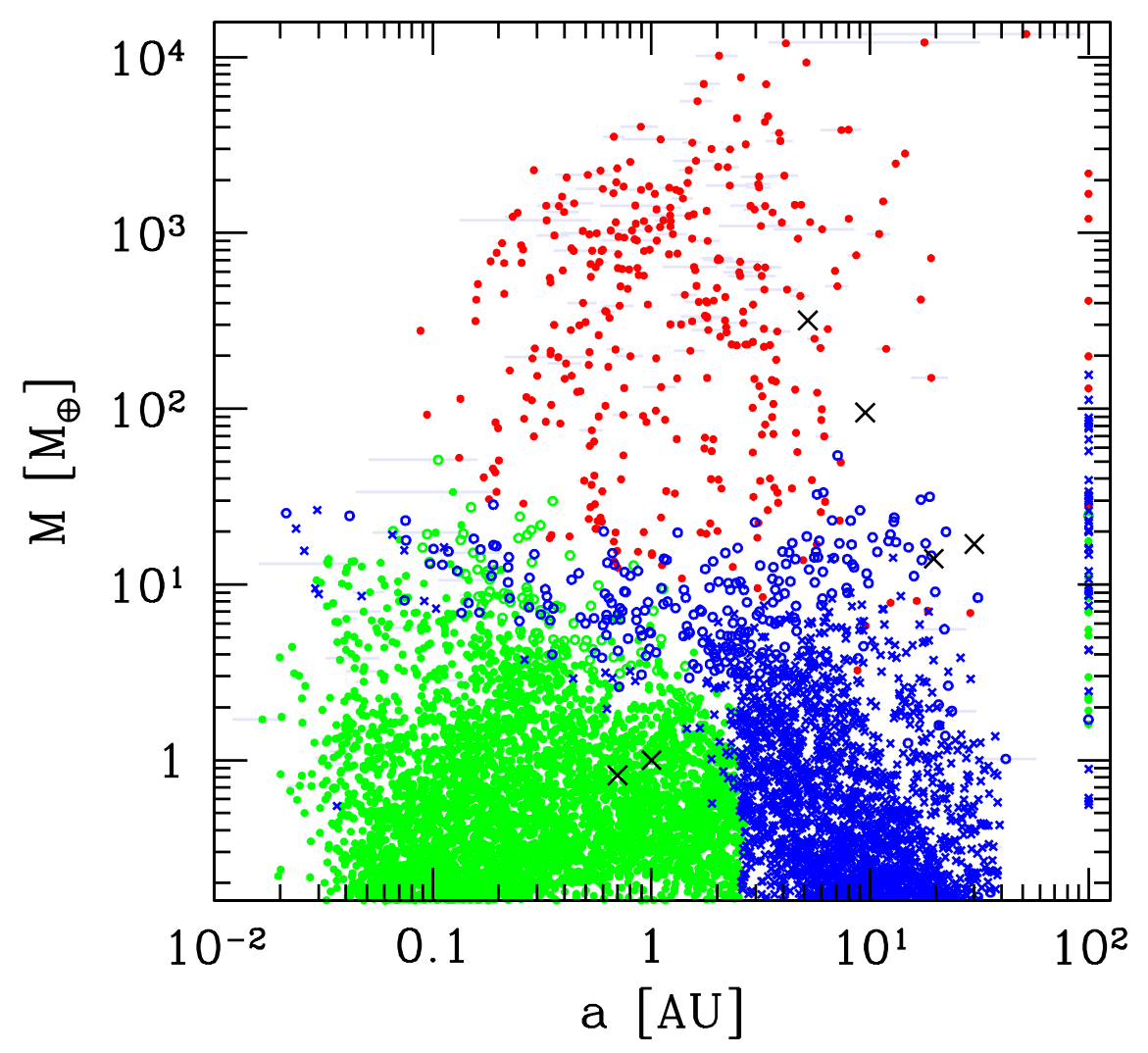

Fig. 8: Synthetic mass-distance diagram. Points shows the semimajor axis, while gray horizontal bar go from $a-e$ to $a+e$. Ejected planets are shown at $100 \mathrm{AU}$. As in Fig. 7, the colors and symbols show the planets' bulk composition. The black crosses represent the solar system planets.

To finally get a statistical overview of the diversity of planetary systems, Figure 8 shows the superposition of all 504 individual systems in the mass-distance plane. This diagram is of similar importance for (extrasolar) planets as the HertzsprungRussell diagram for stars. 
The first, and most fundamental results is that the variation of the initial conditions over a range indicated by observations of protoplanetary disks leads to a high diversity of planetary systems which covers a large part (but not all) of the parameter space that was found to be covered by the observation of extrasolar planets (compare with Fig. 1). The plot also visually highlights the prevalence of low-mass planets that was already a key result of the first population syntheses (Ida \& Lin 2004a). The prevalence is further quantified with the planetary mass function (Sect. The planetary mass function and the distributions of $a, R$ and $L$ ) and the planet frequencies discussed in Sect. Comparison with observations: planet frequencies.

Considering the solar system, we see that the terrestrial planets, Jupiter, and Uranus are in regions that are well populated with synthetic planets, whereas Saturn and Neptune are rather on the outer edge of the populated envelope, at least for the 504 synthetic system shown here. As mentioned, this could be linked to a more compact early configuration of the system as predicted by the Nice Model, where Saturn and Neptune were at about 8 and 14 AU, respectively (Gomes et al. 2005).

For the giant planets, a certain pile-up is see around $1 \mathrm{AU}$, similar as in the observed distribution (Sect. Distributions of planetary properties). It is a consequence of the existence of a preferred formation location for giant planets outside of the water iceline, and a typical inward migration of several (1-10) AU. The finite extent of migration is due to the non-negligible type II migration timescale $(\approx$ viscous timescale), the slowing down in the inner system (Eq. 25), and the typical finite disk lifetimes that are comparable to the formation timescale of the giant planets. This mean that giants often migrate in evolved disk with a mass that has already significantly decreased, slowing down orbital migration (Mordasini et al. 2012a).

In terms of the bulk composition of the core, we see that close-in low-mass planets have an Earth-like composition (green symbols in Fig. 8) as they did not accrete outside of the iceline. But there are also close-in, more massive (sub-)Neptunian planets that have started to form outside of the iceline giving them significant ice mass fractions indicated by blue symbols. They then migrated in through a "horizontal branch" (Mordasini et al. 2009a), as the positive corrotation torque saturates at such masses, leaving only the negative Lindblad torques, which drives fast inward Type I migration. Starting with about 50\% ice in mass in the core while accreting outside of the water iceline, they eventually have an ice mass fraction in the core of about $10-20 \%$, as they accrete rocky planetesimals during their migration through the inner system, such that composition-wise, they are not really Neptune-like. This phenomenon was previously seen in simulations for GJ $436 \mathrm{~b}$ (Figueira et al. 2009). We also see that planets with masses of about $10-30 M_{\oplus}$ have a $\mathrm{H} / \mathrm{He}$ mass fraction of $M_{\text {env }} / M_{\text {core }}=0.1-1$, and more massive planets are giants where $M_{\text {env }} / M_{\text {core }}>1$.

\section{A quite populated planetary desert}

Compared to early population syntheses in particular from the Ida \& Lin model, there is no strong "planetary desert" (absence of intermediate mass planets) visible in Fig. 8, even though a certain dip in the mass function at intermediate masses 
of about 30-100 $M_{\oplus}$ can still be seen in the mass function (Fig. 10). As partially discussed before (Sect. Accretion of gas), the reason is mainly three-fold:

First, in the models shown here, the heating from planetesimal accretion leads to a $\tau_{\mathrm{KH}}$ that decreases less rapidly with increasing mass compared to the Ida \& Lin (2004a) model, as discussed in Sect. Accretion of gas, meaning that planets move less rapidly through the intermediate mass regime. Thus, the probability that the gas disk disappears during this time is higher.

Second, the gas accretion rates obtained in the disk-limited phase calculated with Eq. 13 is often only a few $10^{-4} M_{\oplus} / y$ r. The reason is that cores often only reach a mass sufficient to trigger gas runaway in advanced stages of disk evolution, when the gas surface density has already decreased significantly. Such a timing at first appears unlikely; it is not, if we consider that in most disks, planetary growth is so slow that cores sufficiently massive to trigger gas runaway never form during the gas disk's lifetime (the frequency of stars with giant planets is at most $20 \%$ ). So a late formation is actually probable. This seems to be a difference to pebble-based models (Bitsch et al. 2015b). The removal of disks before cores have a chance to undergo runaway growth also gives naturally rise to a population of numerous super-Earths (Hasegawa \& Pudritz 2012; Alessi et al. 2017).

Third, in contrast to earlier simulations, we find a significant multiplicity of giant planets (see Sect. Comparison with observations: planet frequencies), meaning that individual proto-giants compete for gas while growing, reducing further the maximal gas accretion rates, and leading to more intermediate mass planets.

\section{The $a-R$ distribution}

The results of the Kepler mission (Borucki et al. 2011) has given us a unique insight into the statistics of close, mostly small planets (e.g., Howard et al. 2012; Fressin et al. 2013; Petigura et al. 2013; Mulders et al. 2015; Petigura et al. 2018). As a transit mission, it however yields planetary radii, and not masses, and no unique relation exists that links mass and radius (e.g., Wolfgang et al. 2016). Keeping track of the basic material type that a planet accretes (iron, silicates, ices and $\mathrm{H} / \mathrm{He}$ ) combined with the calculation of the evolution of its internal structure (Eq. 11) make it possible to predict radii from a global model for a direct comparison (Mordasini et al. 2012b).

Fig. 9 shows the synthetic distance-radius diagram at 5 Gyr. In contrast to the mass-distance relation, there is a still signifiant evolution of the radii also after the dissipation of the gas disk because of contraction and atmospheric escape. The plot shows that giant planets with $M_{\text {env }} / M_{\text {core }}>1$ have radii larger than 6-7 $R_{\oplus}$, while intermediate planets with $0.1 \leq M_{\text {env }} / M_{\text {core }} \leq 1$ have radii larger than 3-4 $R_{\oplus}$. To zero order, the planets of intermediate radii have a frequency that is uniform in $\log (R)$. Two prominent feature can be seen:

First, the radii of most giant planets fall in a relatively narrow range of about 10$12.4 R_{\oplus}\left(0.9\right.$ to $1.1 R_{4}$, where $R_{4}$ is the radius of Jupiter). This is expected (Mordasini 


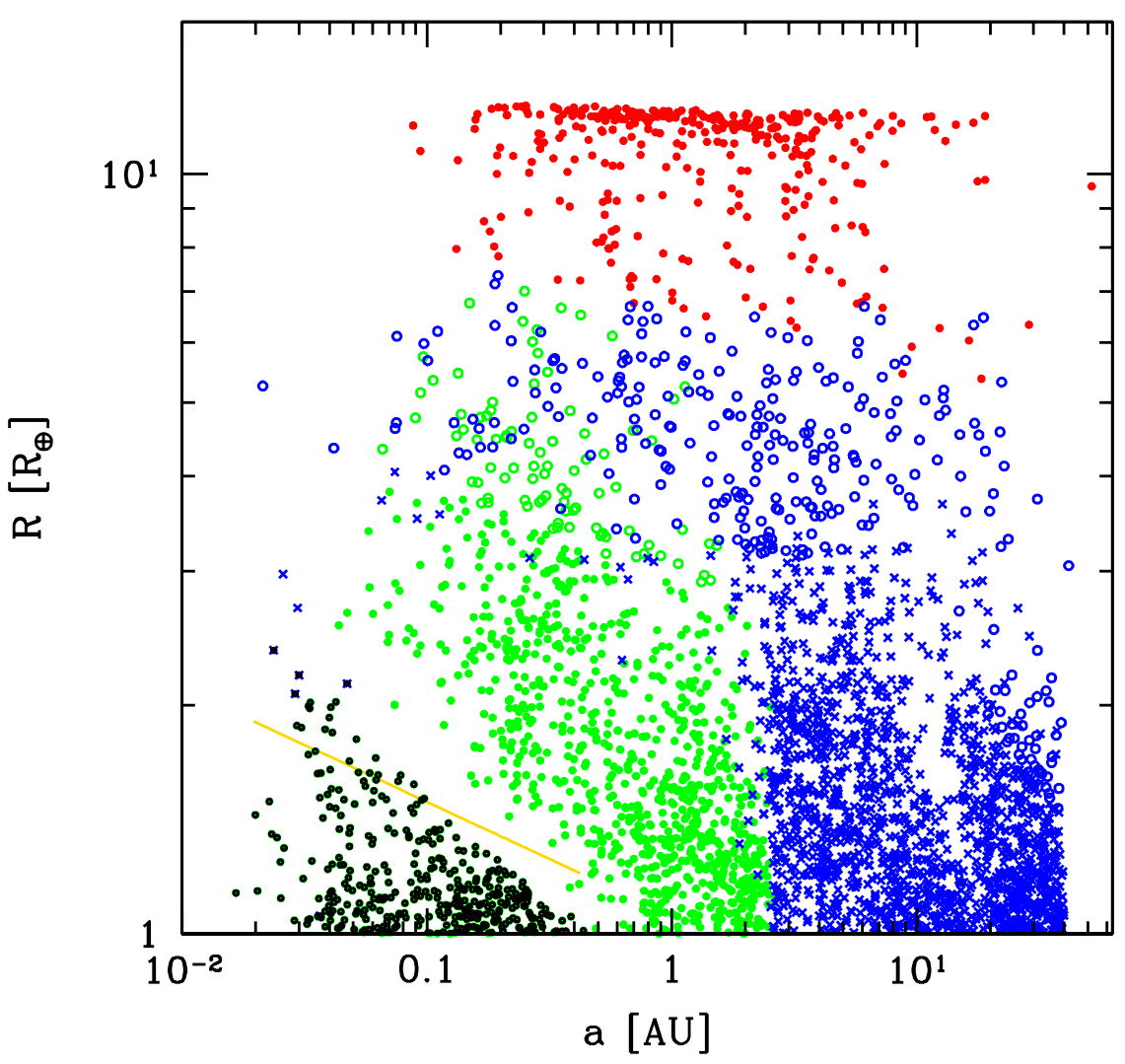

Fig. 9: Synthetic planet radius-semimajor axis diagram at 5 Gyr. The colors and symbols are the same as in Fig. 7. Small black open circles additionally show planets that have lost the entire primordial $\mathrm{H} / \mathrm{He}$ envelope by atmospheric escape. The yellow line shows the location of the gap determined observationally by Van Eylen et al. (2017).

et al. 2012b), as the mass-radius relation of giant planets between about the mass of Saturn and well into the brown dwarf regime is such that the radius is nearly independent of mass, and always around $1 R_{4}$ (Chabrier et al. 2009). The reason is that the interiors become more and more compressible with increasing mass. In the synthetic population here the pile-up is exaggerated as all planets have during evolution the same solar-composition opacity in the atmosphere (Freedman et al. 2014). Varying opacities would cause the radii to vary more (Burrows et al. 2007). Additionally, no bloating effects are included (for an overview, see Baruteau et al. 2016).

A second prominent feature is the gap running diagonally downwards with increasing semimajor axis at small radii. It separates inside of $1 \mathrm{AU}$ planets that have 
kept or lost the primordial $\mathrm{H} / \mathrm{He}$. Close-in low-mass planets lose their envelope because the binding energy of their $\mathrm{H} / \mathrm{He}$ envelope is small compared to the incoming stellar XUV radiation that the planets are exposed to. Note that this evaporation valley was theoretically predicted by models (Owen \& Wu 2013; Lopez \& Fortney 2013) including population synthesis models (Jin et al. 2014) before it was observed (Fulton et al. 2017; Van Eylen et al. 2017). As expected for a simple energy-limited evaporation model with a constant efficiency factor, the model here predicts a slope that is somewhat steeper than observed (Owen \& Wu 2017; Van Eylen et al. 2017). The Earth-like rocky composition of the planets in the region of the gap predicted in the synthesis is consistent with the observed location of the gap (Owen \& Wu 2017; Jin \& Mordasini 2018).

\section{The planetary mass function and the distributions of $a, R$ and $L$}

Figure 10 shows four distributions of fundamental planetary properties. The top left panel shows the mass distribution (including planets at all semimajor axes) P-MF which is also shown with a linear y-axis in Fig. 11. The prediction of the mass function is a key goal of population synthesis. We see a result that is qualitatively similar to earlier results obtained in the one-embryo-per-disk simplification (Mordasini et al. 2009b), and characteristic for the core accretion paradigm: two main regimes exist, one below about $30 M_{\oplus}$ which corresponds to planets with a composition dominated by solids, and one consisting of gas-dominated giant planets at higher masses. The break at around $30 M_{\oplus}$ corresponds to a state when (critical) core and envelope mass are approximately equal, just before gas runaway accretion occurs. The most fundamental aspect of the core accretion paradigm - the existence of a critical core mass - is thus imprinted into the planetary mass function.

In the two regimes below and above $30 M_{\oplus}$ respectively, different physical mechanisms (the accretion of solids vs. the accretion of gas) control the growth of the planets, leading to two different slopes of the mass function. These two basic regimes exist both in earlier syntheses employing the one-embryo-per-disk simplification and in newer ones with numerous concurrently forming protoplanets (as here), explaining why qualitatively, the mass function is similar.

Above $30 M_{\oplus}$, the mass function is to zero approximation flat in $\log (M)$ up to about $5 M_{4}$, i.e., the number $N$ of planets scales with mass $M$ as $N \propto M^{-1}$. Below the break at about $30 M_{\oplus}$, the dependency is steeper, and scales roughly like $N \propto M^{-2}$. Finally, towards the upper end of the planetary mass function above about $5 M_{4}$, the decrease follows a similar steep scaling. These scalings are shown in Fig. 10 with dotted red lines.

The semimajor axis distribution of intermediate and low-mass planets is characterized by a rapid rise in the frequency between 0.01 and $0.1 \mathrm{AU}$, followed by a large interval between 0.1 and almost $10 \mathrm{AU}$ where the distribution is approximately flat (or slightly decreasing) in $\log (a)$. This is similar as indicate by observations (Petigura et al. 2018) for $a \lesssim 1 \mathrm{AU}$. It indicates that despite orbital (Type I) migration 

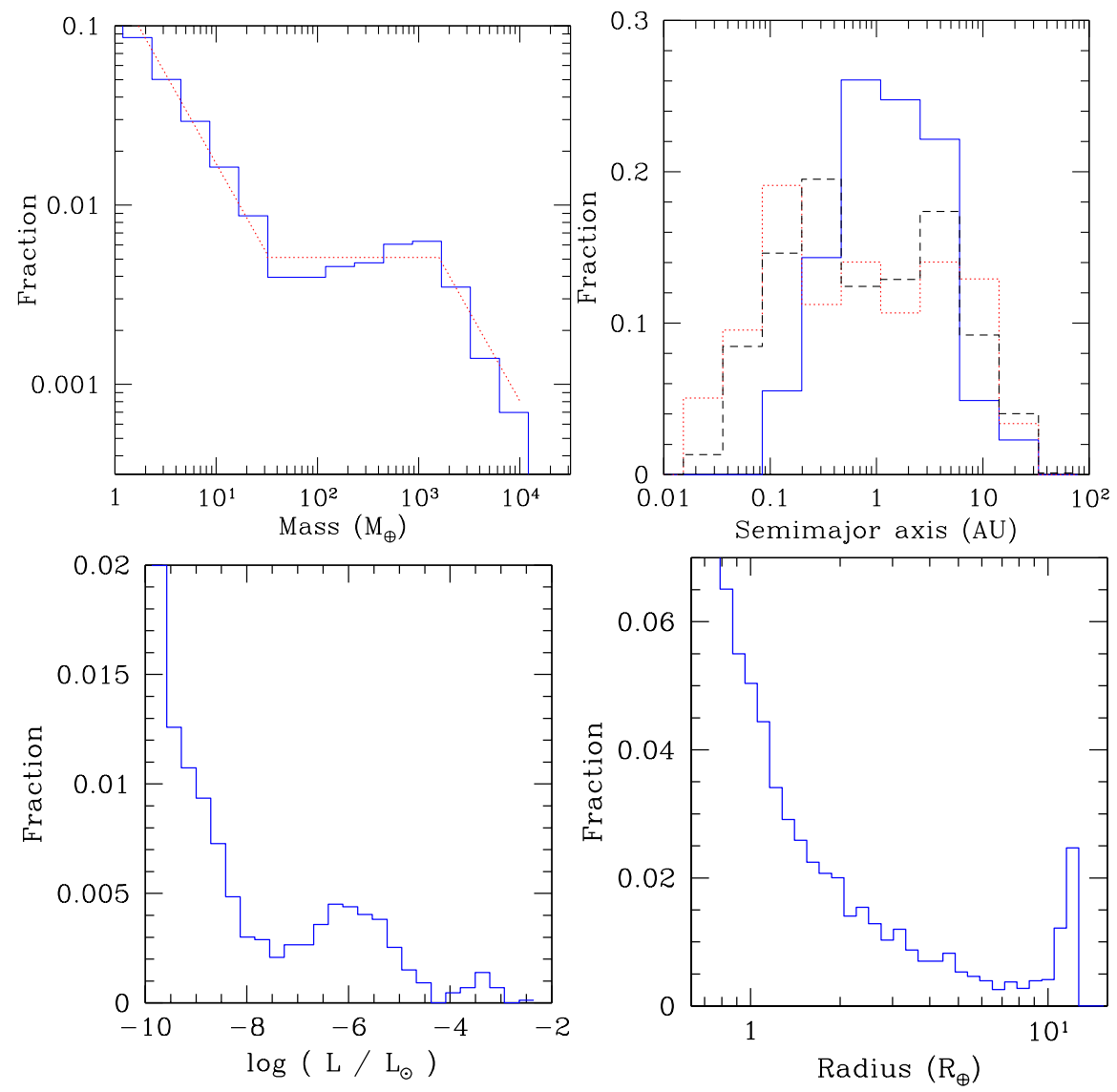

Fig. 10: Distributions of fundamental planetary properties in the synthetic population. Top left: planetary mass function P-MF. The red dotted lines show scalings discussed in the text. Top right: semimajor axis distribution for three mass intervals (blue $>30 M_{\oplus}$; red 10-30 $M_{\oplus}$; black 1-10 $M_{\oplus}$ ). Bottom left: bolometric luminosity. Bottom right: radius. The luminosity is shown at $20 \mathrm{Myr}$, the other panels are for 5 Gyr.

that is included in the model without artificial reduction factors, and which leads for many planets to a significant reduction of the semimajor axis by factors of around 410 or even more relative to the starting position, it nevertheless preserves the initial distribution that is uniform in $\log (a)$ as well. Giant planets are restricted to smaller semimajor axis range (about 0.1 to $6 \mathrm{AU}$ ), with a rapid drop both inside and outside and outside this distance. The distribution peaks a bit inside of $1 \mathrm{AU}$.

The luminosity distribution - shown at $20 \mathrm{Myr}$ - mainly traces the mass distribution (Mordasini et al. 2017) because of the power law relation between mass and luminosity approximately given as $L \propto M^{2}$ at a fixed time. Compared to the mass 
distribution, a third local maximum appears at around $\log \left(L / L_{\odot}\right) \approx-3.5$ which is caused by deuterium burning planets (Mollière \& Mordasini 2012).

The distribution of the radii of the planets (including planets at all semimajor axis) finally is also similar as in simulations using the one-embryo-per-disk simplification (Mordasini et al. 2012b), and contains a local maximum at around 1 Jovian radius (for reasons discussed in Sect. The $a-R$ distribution), and a relatively continuous raise towards smaller radii. This is caused by the prevalence of low-mass planets and the fact that their KH-timescale for gas accretion is long, such that they have small $\mathrm{H} / \mathrm{He}$ mass fractions and thus also small radii (Mordasini et al. 2012b). This KH-timescale effect, and the EOS linking mass, bulk composition, and radius is the same as in the one-embryo-per-disk case, explaining the similarity.

Compared to the input distributions of the starting planetary embryos (initial mass of $0.1 M_{\oplus}$ for all seeds, semimajor axes uniformly distributed in $\log (a)$ between 0.05 and $40 \mathrm{AU}$, all seeds put into the disk at the beginning of the simulation), the final mass and radius distributions are very different, and contain some specific physically explainable sub-structures. This suggest that the specific initial mass of the embryos has not directly influenced these distributions, at least for planets with $M \gg 0.1 M_{\oplus}$. On the other hand, the final semimajor axis distribution is still -to zero order- uniform in $\log (a)$ for planets with masses between 1 and $30 M_{\oplus}$. This could indicate that the input semimajor axis distribution influences this result, and that the final distribution would differ if another initial distribution would be used. Such different distributions seem quite possible, for example because of preferred formation locations of the planetesimals (e.g., Dra̧żkowska et al. 2016), particle pile-ups outside of orbits of already existing planets (e.g., Pinilla et al. 2015) or strong migration traps (e.g., Horn et al. 2012; Hasegawa \& Pudritz 2012). This shows the necessity to include the earlier stages of planet formation in future global models, namely the dust, pebble and planetesimal formation stages.

\section{Comparison with observations: planet frequencies}

Before comparing observed and synthetic distributions, we address the frequency of three fundamental planet types predicted by the synthesis: first, giant planets with a mass of at least $300 M_{\oplus}$, second close-in planets (period $\leq 100$ d, i.e., $a \leq 0.42 \mathrm{AU}$, radius $\geq 1 R_{\oplus}$ ) comparable to the planets probed by Kepler (e.g., Marcy et al. 2014; Petigura et al. 2018), and third planets in the classical habitable zone with mass of 0.3 to $5 M_{\oplus}$ and a semimajor axis of 0.95 to $1.37 \mathrm{AU}$ (Kasting et al. 1993). We give the overall fraction of stars having such planets, indicating also their multiplicity. For the comparison with observed frequencies (Sect. Frequencies of planet types), one should keep in mind that the synthetically predicted absolute frequencies are less robust than the relative ones. The reason is that the absolute frequencies depend more directly on model parameters like the arbitrary chosen planetesimal size of 300 $\mathrm{m}$ which influences the solid accretion rate, as discussed in Sect. Accretion of solids. 
Assuming a smaller (bigger) size of the planetesimals would increase (decrease) the fraction of disks in which massive planets form.

\begin{tabular}{|c|c|c|c|}
\hline $\mathrm{N}$ & Giant planets & Close-in planets & Planets in $\mathrm{HZ}$ \\
\hline 1 & 4.8 & 8.4 & 30.7 \\
\hline 2 & 7.4 & 12.8 & 8.2 \\
\hline 3 & 5.4 & 11.4 & 1.0 \\
\hline 4 & 0.4 & 10.0 & 0.0 \\
\hline$\geq 5$ & 0.0 & 11.4 & 0.0 \\
\hline Overall synthetic & 18.0 & 54.0 & 39.9 \\
\hline Overall observed & $10-20$ & $50-60$ & unknown \\
\hline
\end{tabular}

Table 1: Percentage of stars with $\mathrm{N}$ planets of the given type in the synthetic population, and comparison to observations (last line).

We see that the overall fraction of stars with giant planets is about $18 \%$. The most frequent number of giant planets per star is 2, occurring for $7.4 \%$ of all stars. Single giant planets and stars with 3 giants are both on the 5\% level. Only $0.4 \%$ of the stars have 4 giant planets, and none has more than that. Note that these numbers may be upper limits, as the calculation of the N-body interaction was stopped at $10 \mathrm{Myr}$, such that we do not take into account ejections or collisions at later moments. The overall frequency of giant planets is however similar as observed (10-20\%, see Sect. Frequencies of planet types), even if the multiplicity in the synthetic population might be higher than observed (Bryan et al. 2016).

The population of close-in planets is dominated by small (or low-mass) planets as visible from Figs. 8 and 9. Even if a quantitative comparison with the HARPS high-precision RV survey or the Kepler transit survey would require a dedicated modeling of the observational biases (e.g., Mayor et al. 2011; Petigura et al. 2018), the frequencies of these close-in planets in Table 1 makes nevertheless clear that such planets are a very common outcome of the formation process, similarly as observed (Sect. Frequencies of planet types). About $54 \%$ of the synthetic systems have such planets which is similar to the observed frequency (Petigura et al. 2013), and the multiplicity is high, with a mean number of about 3 such planets per star that has this type of planet, which is again at least qualitatively similar as observed. This is high frequency of close-in planets can only be reproduced in the syntheses if a steep, centrally concentrated distribution of the planetesimals is used, as described in Sect. Initial conditions and parameters (see also Chiang \& Laughlin 2013). This is an interesting constraint for drift and planetesimal formation models (e.g., Drążkowska et al. 2016).

The fraction of stars with planets in the classical habitable zone is in contrast lower, but with $39.9 \%$ still very significant. The mean number of this type of planet is 1.25 for those stars that have such planets, i.e. typically there is only one planet in the classical habitable zone per such system. Observationally, the frequency of solarlike stars with potentially habitable planets is still now well known, with estimates 
ranging from 1 to $100 \%$ (e.g., Burke et al. 2015). For M-dwarfs, where a direct determination of this frequency is in contrast already possible with radial velocity surveys, the fraction is $0.41_{-0.13}^{+0.54}$ (Bonfils et al. 2013).

\section{Comparison with observations: distributions}

Figure 11 compares the synthetic mass and radius distribution with their observational counterparts as found by the HARPS high precision RV survey (Mayor et al. 2011) and an analysis of the Kepler transit survey (Howard et al. 2012). The observed distributions are corrected for the observational bias. The synthetic distributions only include the planets in the same mass/radius and orbital distance range as in the observational samples.
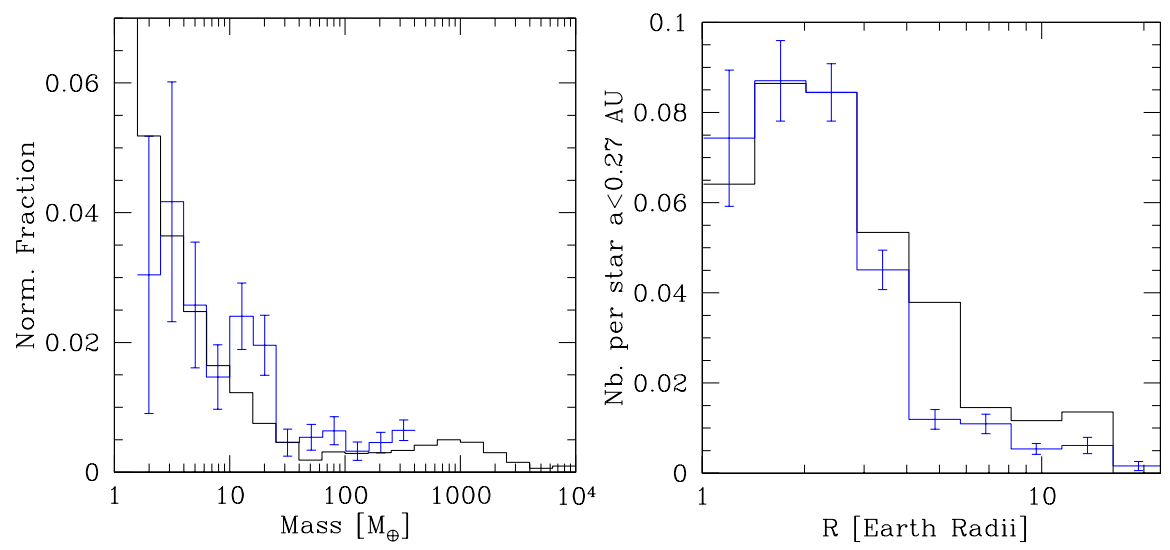

Fig. 11: Comparison of the synthetic mass and radius distribution (black) of the population introduced at the beginning of Sect. Results with the bias-corrected observed distributions (blue) of the HARPS high precision RV survey (Mayor et al. 2011) and the Kepler transit survey (Howard et al. 2012). The two theoretical and observed distributions were normalized to the same value at the bin at $30 M_{\oplus}$ and 2-3 $R_{\oplus}$, respectively.

The basic shape echoes the distributions that include all synthetic planets shown in Fig. 10. The synthetic mass distribution compares quite well with the observed one, in particular regarding the aforementioned break in the mass function at about $30 M_{\oplus}$ and the associated change of the slope that was already predicted in early population syntheses (Mordasini et al. 2009b). This break is visible also in other bias-corrected high-precision RV surveys like Howard et al. (2010), and even in the biased, directly observed mass distribution (Schneider et al. 2011; Wright et al. 2011). The log-flat distribution between about $30 M_{\oplus}$ and $5 M_{4}$ agrees with the ob- 
served distribution (Marcy et al. 2005) as well. The fact that the two theoretically predicted slopes are visible also in the observed distribution, and that the break occurs at a similar mass in theory and observations constitutes a major success of the core accretion theory, and planet formation theory in general. This is of an astrophysical importance comparable to the development of a theory for the stellar initial mass function (Chabrier 2003), including the classical Salpeter slope (Salpeter 1955).

In the radius distribution of synthetic planets inside of $0.27 \mathrm{AU}$, a significant difference is seen for the peak at about 1 Jovian radius relative to the distribution that includes all orbital distance in Fig. 10, as already found in Mordasini et al. (2012b): the peak is less pronounced compared to Fig. 10 because of the broader bins, and due to the fact that only planets inside of $0.27 \mathrm{AU}$ are included (as in the observational sample), while synthetic giant planets are mostly further out (see Fig. 9). In these broad bins, the evaporation valley and the associated gap in the radius distribution is not visible as a finer radius resolution is required (Jin \& Mordasini 2018). Another difference is that the synthetic distribution is less abruptly increasing towards the small radii compared to the observed one.

We note that the population contains many planets with masses still lower than 1 $M_{\oplus}$ and/or radii less than $1 R_{\oplus}$. They are found in systems in which not much growth and migration occurred during the first $10 \mathrm{Myr}$ (the time during which the systems' formation was simulated as explained in Sect. Initial conditions and parameters). At $10 \mathrm{Myr}$, there are still around 20 low-mass protoplanets left. Such systems arise for initial conditions with low amounts of solids $($ low $[\mathrm{Fe} / \mathrm{H}]$ and/or initial gas disk masses) and/or short disk lifetimes (see Mordasini et al. 2012a for an extensive discussion of the correlations between disk and planetary properties). This dependency is illustrated by the systems forming at low $[\mathrm{Fe} / \mathrm{H}]$ in Fig. 7 .

Over longer timescales, these low-mass planets could collide to form more massive planets, such that this result could be an artifact of only modeling the N-body interaction during $10 \mathrm{Myr}$. This also means that all results concerning planets with masses close to the initial embryo mass should be taken with caution.

\section{Correlations with disk properties}

An important application of population synthesis is to understand how the planetary formation process depends on the properties of the protoplanetary disk. The most important observed correlation in this context is that the probability of observing giant planets increases with the host star metallicity (e.g., Gonzalez 1997; Santos et al. 2004; Fischer \& Valenti 2005), the so-called "metallicity effect", as discussed in Sect Correlations with stellar properties. Syntheses by Ida \& Lin (2004b) and later Mordasini et al. (2009b, 2012a) have shown quantitatively that this metallicity effect is a natural outcome of the core accretion model. This is under the assumption that stellar and disk metallicity are proportional (Sect. Initial conditions and parameters), and further, that the surface density of planetesimals increases with the mass fraction 
of heavy elements as well (Eq. 5), which is indicated by planetesimal formation models (Brauer et al. 2008). In this case, the growth of a planetary core by accreting planetesimals occurs on a shorter timescale (Eq. 10), and leads to more massive cores as well (Kokubo \& Ida 2012). Thus, there is a higher chance to grow to the critical core mass and trigger rapid gas accretion before the gas disk has dissipated.

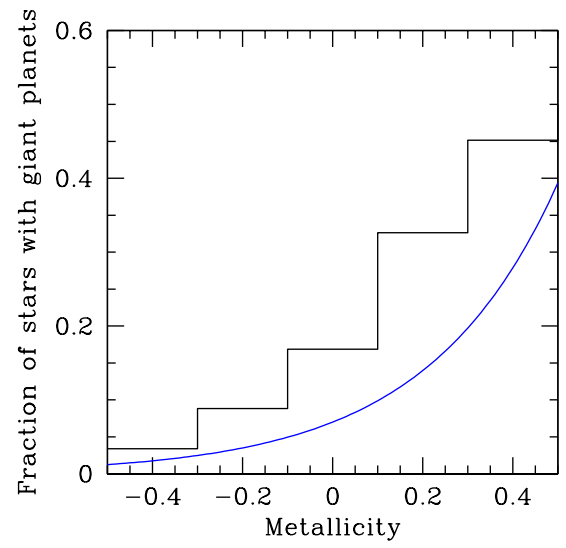

Fig. 12: Fraction of stars with giant planets $\left(M \geq 300 M_{\oplus}\right)$ as found in the synthetic population (black line). The blue line shows the fit to the observed frequency from Mortier et al. (2013).

Figure 12 shows the fraction of stars with at least one giant planet (mass higher then $300 M_{\oplus}$ ) in the present synthesis for $1 M_{\odot}$ stars as a function of metallicity, compared to a fit to the observed relation by Mortier et al. (2013). While the model over-predicts the number of giant planets in absolute terms, it agrees with the observed relative increase quite well.

Further correlation of disk properties were studied by Mordasini et al. (2012a) where - not surprisingly - a high number of correlations was found: for example, for the planetary initial mass function, high metallicities lead to a higher frequency of giant planets while higher initial disk (gas) masses lead mainly to giant planets of a higher mass. For long disk lifetimes, giant planets are both more frequent and massive. At low metallicities, very massive giant planets cannot form, but otherwise giant planet mass and metallicity are nearly uncorrelated. In contrast, (maximum) planet masses and disk gas masses are correlated.

\section{Testing theoretical sub-models}

The final goal of population synthesis is to improve our understanding of planet formation and evolution. For this task, specific sub-models are put via syntheses 
to the observational test as described in Sect. Workflow of the population synthesis method. A non-conclusive list of mechanisms that were addressed in this way is (1) orbital migration, mostly (non)isothermal Type I migration. Early population syntheses (Ida \& Lin 2008a; Mordasini et al. 2009b) showed that the observed distribution of planetary orbital distances and the fraction of stars with hot Jupiters can only be reproduced if the migration rates predicted by the then existing (isothermal) type I migration models (Tanaka et al. 2002) are strongly reduced. This sparked numerous dedicated studies that led to more realistic non-isothermal type I migration rates (like Masset \& Casoli 2010; Kley et al. 2009; Paardekooper et al. 2010). These new models where then in turn included in the population syntheses, leading to orbital distances that are more similar to observations (Dittkrist et al. 2014). This is a prime example of how population synthesis and specialized models advance each other. (2) grain dynamics and opacities in protoplanetary atmospheres influencing the bulk composition of planets. Here it was found with population syntheses that the observed mass-radius relation (i.e., the bulk composition) of extrasolar planets with $\mathrm{H} / \mathrm{He}$ can be reproduced only if the grain opacity in protoplanetary atmospheres is clearly lower than the ISM opacity (Mordasini et al. 2014). This led to the development of specialized models for the grain dynamics (growth, settling, destruction) and resulting opacities (Ormel 2014; Mordasini 2014), which are indeed low compared to the ISM. (3) disk inhomogeneities and transitions leading to migration traps (Hasegawa \& Pudritz 2011; Hasegawa \& Pudritz 2013; Coleman \& Nelson 2016), (4) stellar cluster environments (Ndugu et al. 2018), (5) the gas accretion shock structure and the luminosity of young giant planets (Mordasini et al. 2017), (6) constraints on formation pathways from the chemical composition and atmospheric spectra (e.g., Marboeuf et al. 2014; Madhusudhan et al. 2014), or finally (7) atmospheric escape of primordial H/He envelopes (Jin et al. 2014; Jin \& Mordasini 2018).

\section{Predictions: observational confirmations and rejections}

Another central application of population syntheses are predictions for upcoming instruments and surveys, i.e., quantitative theoretical predictions that can be falsified with more accurate observational methods. Mordasini et al. (2009b) for example studied the consequences for the fraction of stars with detectable planets and the shape of the P-MF if the radial velocity measurement accuracy improves from 10 $\mathrm{m} / \mathrm{s}$ to $1 \mathrm{~m} / \mathrm{s}$ and $0.1 \mathrm{~m} / \mathrm{s}$.

Some of the predictions made by population syntheses were later confirmed by observations, others turned out to be inconsistent. Some of the most important confirmed predictions are: (1) the prevalence of low-mass and small planets (Ida \& Lin 2004b) that was made well before they were found by precise RV surveys and Kepler (e.g., Howard et al. 2010; Mayor et al. 2011; Borucki et al. 2011), (2) the break in the planetary mass function at around $30 M_{\oplus}$ (Mordasini et al. 2009b) that was later detected by Howard et al. (2010); Mayor et al. (2011) through high-precision 
$\mathrm{RV}$, (3) the pile-up of planetary radii around $1 R_{4}$ (Mordasini et al. 2012b) which was not visible in early polluted Kepler data but which is has become apparent recently in cleaned samples (Petigura et al. 2018), or (4) together with other models (Owen \& Wu 2013; Lopez \& Fortney 2013), the depleted evaporation valley in the distance-radius plane (Jin et al. 2014), which was later confirmed observationally by Fulton et al. (2017) and Van Eylen et al. (2017).

Important predictions that turned out to be inconsistent with observations were (1) the existence of a strongly depleted "planetary desert" (Ida \& Lin 2004b), i.e., a strong depletion in the frequency of planets with masses between 10 to $100 M_{\oplus}$ (a weak depletion might actually exist, see Fig. 1), or (2) an absence of close-in planets $\left(a \lesssim 0.1 \mathrm{AU}\right.$ ) with masses less than approximately $10 M_{\oplus}$ (Mordasini et al. 2009a). These inconsistent predictions are actually of particular interest, as they point at important shortcomings in the theoretical models. In the former case, the gas accretion rate in the runaway phase was overestimated (see Sect. Accretion of gas and A quite populated planetary desert for limiting effects), in the latter, a strongly reduced isothermal type I migration rate and a criterion for the transition into type II migration based only on the thermal criterion caused the discrepancy. These shortcomings were then addressed in later generations of the models (Sect. Overview of population synthesis models in the literature), and helped in this way to improve the understanding of the planet formation process, and to avoid oversimplifications.

\section{Summary and conclusions}

The increase in statistical observational constraints on extrasolar planets has been enormous in the last two decades. Both ground and space-based surveys have derived distributions of fundamental planetary properties like the frequency of planets in the mass-distance and radius-distance planes, the planetary mass function, the eccentricity distribution, or the planetary mass-radius relation.

All these observed distributions put strong statistical constraints on the theory of planet formation and evolution (Sect. Statistical observational constraints). The method of choice to use these constraints in order to improve our understanding of planet formation is populations synthesis, an approach that has been used for many decades in various fields of stellar astrophysics.

The underlying idea of population synthesis is that the same physical processes govern the formation of planets in all protoplanetary disk, but that the initial conditions for these processes (the properties of the parent protoplanetary disk) and potentially the boundary conditions (like the stellar cluster environment) differ, and that this gives raise to the observed diversity of (extrasolar) planets.

Methodically, population syntheses (Sect. Population synthesis method) thus consist of two main components: first, probability distributions of the initial conditions (disk properties, Sect. Initial conditions and parameters) and second a global end-to-end model of planet formation and evolution that can predict observable planetary properties based directly on disk properties. Building both on the core 
accretion and the gravitational instability scenario, the different modern population synthesis models in the literature (Sect. Overview of population synthesis models in the literature) include in a self-consistently coupled way an impressive number of physical processes like the evolution of the protoplanetary disks of solids and gas, planetary accretion of solids (both of planetesimals and pebbles), the accretion of gas, orbital migration, and N-body interactions, and often several more. A description of these sub-models can be found in Sect. Global models: simplified but linked.

The sub-models describing these processes are, however, either parameterized or low-dimensional static approximations of 3D dynamical systems (Sect. Lowdimensional approximation). To what extent the dynamical multi-dimensional nature of one individual governing process (like orbital migration or pebble accretion) can be "distilled" into a simpler, lower-dimensional approximation that still captures the essence of the physics while allowing population syntheses with acceptable computational costs, is a key challenge for any population synthesis approach, and an ongoing development.

On the other hand, population synthesis is often the only possibility to observationally test theoretical models of a specific mechanism (Sect. Testing theoretical sub-models) as it produces synthetic data that can be directly statistically compared with observations, factoring in the non-linear interaction between the different mechanism concurrently acting during planet formation. This leads to planetary formation tracks and synthetic planetary systems of a large diversity (Sect. Diversity of planetary system architectures). Population synthesis therefore also has a high predictive power that is difficult to achieve with theoretical models of just one physical process.

In the Section Results, the typical output obtained from a population synthesis model is presented and linked to underlying physical effects. These are the distribution of planets in the mass-distance and radius-distance plane, the planetary mass function, and the distribution of radii, orbital distances, and luminosities. These distributions are compared with their observed counterparts (Sect. Comparison with observations: distributions).

Among these results, a first key prediction of population synthesis models (Sect. The $a-M$ distribution) was the prevalence of low-mass and small planets that was later observationally confirmed by high-precision radial velocity surveys and the Kepler satellite (Sect. Predictions: observational confirmations and rejections). Clearly this has important implications beyond planet formation theory like for the question about the existence of other habitable planets.

A second key prediction was the existence of two regimes in the planetary mass function (Sect. The planetary mass function and the distributions of $a, R$ and $L$ ) with a break at around $30 M_{\oplus}$ when the transition from solid to gas-dominated planet occurs at the critical core mass. The most fundamental aspect of the core accretion paradigm - the existence of a critical core mass - is thus imprinted into the planetary mass function. This prediction was later confirmed by high-precision RV surveys (Sect. Predictions: observational confirmations and rejections). The finding that the two theoretically predicted slopes in the mass function are visible also in 
the observed distribution, and that the observed break occurs at a similar mass as theoretically predicted represents a major success for the core accretion theory, and of planet formation theory in general. This is in a broader astrophysical context of similar importance as the development of a theory for the stellar initial mass function.

A third, maybe even more fundamental insight is that the population syntheses show that the variation of the initial conditions over a range suggested by protoplanetary disk observations lead to an extreme diversity in the resulting synthetic planetary systems, which is probably the single most characteristic property also of the actual extrasolar planet population. It indicates that at least some of the strong non-linearities and feedback mechanisms occurring during planet formation are indeed captured in the theoretical models.

These points do, however, clearly not mean that the current population models describe in a definitive way the actual planet formation and evolution process. They rather reflect the state of the field of planet formation theory where important physical mechanisms governing planet formation are still not well understood (Sect. Confronting theory and observation). It is therefore not surprising that other important predictions made by population synthesis models turned out to be inconsistent with observations (Sect. Predictions: observational confirmations and rejections). But it is exactly the quantitative falsifability of population synthesis that is important, as this it is the key to reject some theoretical concepts or to identify missing ones, improving in this way our understanding of how planets form and evolve.

Acknowledgements: C.M. thanks Ralph Pudritz for the invitation to write this review and for the editorial guidance. C.M. acknowledges the support from the Swiss National Science Foundation under grant BSSGI0_155816 "PlanetsInTime". Parts of this work have been carried out within the frame of the National Center for Competence in Research PlanetS supported by the SNSF.

\section{References}

Alessi, M., Pudritz, R. E., \& Cridland, A. J. 2017, MNRAS, 464, 428

Alexander, R., Pascucci, I., Andrews, S., Armitage, P., \& Cieza, L. 2014, Protostars and Planets VI, 475

Alexander, R. D. \& Armitage, P. J. 2009, ApJ, 704, 989

Alibert, Y., Carron, F., Fortier, A., et al. 2013, A\&A, 558, A109

Alibert, Y., Mordasini, C., \& Benz, W. 2004, A\&A, 417, L25

Alibert, Y., Mordasini, C., Benz, W., \& Winisdoerffer, C. 2005, A\&A, 434, 343

Andrews, S. M., Wilner, D. J., Hughes, A. M., Qi, C., \& Dullemond, C. P. 2010, ApJ, 723, 1241

Bai, X.-N. 2016, ApJ, 821, 80

Baruteau, C., Bai, X., Mordasini, C., \& Mollière, P. 2016, Space Sci.Rev., 205, 77

Baruteau, C. \& Masset, F. S. 2008, ApJ, 672, 1054

Benítez-Llambay, P., Masset, F., Koenigsberger, G., \& Szulágyi, J. 2015, Nature, 520, 63

Benz, W., Ida, S., Alibert, Y., Lin, D., \& Mordasini, C. 2014, Protostars and Planets VI, 691

Birnstiel, T. \& Andrews, S. M. 2014, ApJ, 780, 153

Birnstiel, T., Klahr, H., \& Ercolano, B. 2012, A\&A, 539, 148 
Bitsch, B., Johansen, A., Lambrechts, M., \& Morbidelli, A. 2015a, A\&A, 575, A28

Bitsch, B., Lambrechts, M., \& Johansen, A. 2015b, A\&A, 582, A112

Bodenheimer, P. H., Hubickyj, O., \& Lissauer, J. J. 2000, Icarus, 143, 2

Bodenheimer, P. H. \& Pollack, J. B. 1986, Icarus, 67, 391

Bonfils, X., Delfosse, X., Udry, S., et al. 2013, A\&A, 549, A109

Borucki, W. J., Koch, D. G., Basri, G., et al. 2011, ApJ, 728, 117

Boss, A. P. 1995, Science, 267, 360

Boss, A. P. 1997, Science, 276, 1836

Bowler, B. P. 2016, PASP, 128, 102001

Brauer, F., Dullemond, C. P., \& Henning, T. 2008, A\&A, 480, 859

Bruzual, G. \& Charlot, S. 2003, MNRAS, 344, 1000

Bryan, M. L., Knutson, H. A., Howard, A. W., et al. 2016, ApJ, 821, 89

Burke, C. J., Christiansen, J. L., Mullally, F., et al. 2015, ApJ, 809, 8

Burrows, A., Hubeny, I., Budaj, J., \& Hubbard, W. B. 2007, ApJ, 661, 502

Cameron, A. G. W. 1978, Moon and Planets, 18, 5

Casoli, J. \& Masset, F. S. 2009, ApJ, 703, 845

Cassan, A., Kubas, D., Beaulieu, J., \& al. 2012, Nature, 481, 167

Chabrier, G. 2003, The Publications of the Astronomical Society of the Pacific, 115, 763

Chabrier, G., Baraffe, I., Leconte, J., Gallardo, J., \& Barman, T. S. 2009, Cool Stars, 1094, 102

Chambers, J. E. 1999, MNRAS, 304, 793

Chambers, J. E. 2009, ApJ, 705, 1206

Chiang, E. \& Laughlin, G. 2013, MNRAS accepted

Cimerman, N. P., Kuiper, R., \& Ormel, C. W. 2017, MNRAS, 471, 4662

Coleman, G. A. L. \& Nelson, R. P. 2014, MNRAS, 445, 479

Coleman, G. A. L. \& Nelson, R. P. 2016, MNRAS, 457, 2480

Coleman, G. A. L., Papaloizou, J. C. B., \& Nelson, R. P. 2017, MNRAS, 470, 3206

Coughlin, J. L., Mullally, F., Thompson, S. E., et al. 2016, ApJS, 224, 12

Cresswell, P. \& Nelson, R. P. 2008, A\&A, 482, 677

Crida, A., Morbidelli, A., \& Masset, F. S. 2006, Icarus, 181, 587

Cridland, A. J., Pudritz, R. E., \& Alessi, M. 2016, MNRAS, 461, 3274

Cridland, A. J., Pudritz, R. E., Birnstiel, T., Cleeves, L. I., \& Bergin, E. A. 2017, ArXiv e-prints

Cumming, A., Butler, R. P., Marcy, G. W., et al. 2008, PASP, 120, 531

D'Angelo, G. \& Lubow, S. H. 2008, ApJ, 685, 560

David, T. J., Hillenbrand, L. A., Petigura, E. A., et al. 2016, Nature, 534, 658

Dell'Omodarme, M., Valle, G., Degl'Innocenti, S., \& Prada Moroni, P. G. 2012, A\&A, 540, A26

Dittkrist, K.-M., Mordasini, C., Klahr, H., Alibert, Y., \& Henning, T. 2014, A\&A, 567, A121

Donati, J. F., Moutou, C., Malo, L., et al. 2016, Nature, 534, 662

Drążkowska, J., Alibert, Y., \& Moore, B. 2016, A\&A, 594, A105

Duffell, P. C., Haiman, Z., MacFadyen, A. I., D’Orazio, D. J., \& Farris, B. D. 2014, ApJ, 792, L10

Dürmann, C. \& Kley, W. 2015, A\&A, 574, A52

Fabrycky, D. C. \& Tremaine, S. 2007, ApJ, 669, 1298

Figueira, P., Pont, F., Mordasini, C., et al. 2009, A\&A, 493, 671

Fischer, D. A. \& Valenti, J. A. 2005, ApJ, 622, 1102

Forgan, D. \& Rice, K. 2013, MNRAS, 432, 3168

Forgan, D. H., Hall, C., Meru, F., \& Rice, W. K. M. 2018, MNRAS, 474, 5036

Fortier, A., Alibert, Y., Carron, F., Benz, W., \& Dittkrist, K.-M. 2013, A\&A, 549, 44

Fouchet, L., Alibert, Y., Mordasini, C., \& Benz, W. 2012, A\&A, 540, 107

Freedman, R. S., Lustig-Yaeger, J., Fortney, J. J., et al. 2014, ApJS, 214, 25

Fressin, F., Torres, G., Charbonneau, D., et al. 2013, ApJ, 766, 81

Fulton, B. J., Petigura, E. A., Howard, A. W., et al. 2017, AJ, 154, 109

Goldreich, P. \& Tremaine, S. 1979, ApJ, 233, 857

Gomes, R., Levison, H. F., Tsiganis, K., \& Morbidelli, A. 2005, Nature, 435, 466

Gonzalez, G. 1997, MNRAS, 285, 403

Greenzweig, Y. \& Lissauer, J. L. 1992, Icarus, 100, 440 
Haisch, K. E., Lada, E. A., \& Lada, C. J. 2001, ApJ, 553, L153

Hasegawa, Y. \& Pudritz, R. E. 2011, MNRAS, 417, 1236

Hasegawa, Y. \& Pudritz, R. E. 2012, ApJ, 760, 117

Hasegawa, Y. \& Pudritz, R. E. 2013, ApJ, 778, 78

Hayashi, C. 1981, Progress of Theoretical Physics Supplement, 70, 35

Hellary, P. \& Nelson, R. P. 2012, MNRAS, 419, 2737

Horn, B., Lyra, W., Mac Low, M.-M., \& Sándor, Z. 2012, ApJ, 750, 34

Howard, A. W., Marcy, G. W., Bryson, S. T., et al. 2012, ApJS, 201, 15

Howard, A. W., Marcy, G. W., Johnson, J. A., et al. 2010, Science, 330, 653

Hueso, R. \& Guillot, T. 2005, A\&A, 442, 703

Ida, S. \& Lin, D. N. C. 2004a, ApJ, 604, 388

Ida, S. \& Lin, D. N. C. 2004b, ApJ, 616, 567

Ida, S. \& Lin, D. N. C. 2008a, ApJ, 673, 487

Ida, S. \& Lin, D. N. C. 2008b, ApJ, 685, 584

Ida, S. \& Lin, D. N. C. 2010, ApJ, 719, 810

Ida, S., Lin, D. N. C., \& Nagasawa, M. 2013, ApJ, 775, 42

Ida, S. \& Makino, J. 1993, Icarus, 106, 210

Ikoma, M., Nakazawa, K., \& Emori, H. 2000, ApJ, 537, 1013

Jin, S. \& Mordasini, C. 2018, ApJ, 853, 163

Jin, S., Mordasini, C., Parmentier, V., et al. 2014, ApJ, 795, 65

Johansen, A. \& Lambrechts, M. 2017, Annual Review of Earth and Planetary Sciences, 45, 359

Jurić, M. \& Tremaine, S. 2008, ApJ, 686, 603

Kasting, J. F., Whitmire, D. P., \& Reynolds, R. T. 1993, Icarus, 101, 108

Kley, W., Bitsch, B., \& Klahr, H. 2009, A\&A, 506, 971

Kley, W. \& Nelson, R. P. 2012, ARA\&A, 50, 211

Kokubo, E. \& Ida, S. 2000, Icarus, 143, 15

Kokubo, E. \& Ida, S. 2002, ApJ, 581, 666

Kokubo, E. \& Ida, S. 2012, Progress of Theoretical and Experimental Physics, 2012

Kornet, K., Stepinski, T. F., \& Rozyczka, M. 2001, A\&A, 378, 180

Kozai, Y. 1962, AJ, 67, 591

Kretke, K. A. \& Lin, D. N. C. 2012, ApJ, 755, 74

Kuiper, G. P. 1951, Proceedings of the National Academy of Science, 37, 1

Lambrechts, M. \& Johansen, A. 2012, A\&A, 544, 32

Lambrechts, M. \& Johansen, A. 2014, A\&A, 572, A107

Levison, H. F., Thommes, E. W., \& Duncan, M. J. 2010, ApJ, 139, 1297

Lin, D. N. C. \& Papaloizou, J. C. B. 1986a, ApJ, 307, 395

Lin, D. N. C. \& Papaloizou, J. C. B. 1986b, ApJ, 309, 846

Lodders, K. 2003, ApJ, 591, 1220

Lopez, E. D. \& Fortney, J. J. 2013, ApJ, 776, 2

Lubow, S. H., Seibert, M., \& Artymowicz, P. 1999, ApJ, 526, 1001

Lüst, R. 1952, Zeitschrift Naturforschung Teil A, 7, 87

Lynden-Bell, D. \& Pringle, J. E. 1974, MNRAS, 168, 603

Lyra, W., Paardekooper, S.-J., \& Mac Low, M.-M. 2010, ApJ, 715, L68

Madhusudhan, N., Amin, M. A., \& Kennedy, G. M. 2014, ApJ, 794, L12

Mamajek, E. E. 2009, in American Institute of Physics Conference Series, ed. T. Usuda, M. Tamura, \& M. Ishii, Vol. 1158, 3-10

Manara, C. F., Rosotti, G., Testi, L., et al. 2016, A\&A, 591, L3

Mann, A. W., Newton, E. R., Rizzuto, A. C., et al. 2016, AJ, 152, 61

Marboeuf, U., Thiabaud, A., Alibert, Y., Cabral, N., \& Benz, W. 2014, A\&A, 570, A36

Marcy, G. W., Butler, R. P., Fischer, D. A., et al. 2005, Progress of Theoretical Physics Supplement, 158

Marcy, G. W., Weiss, L. M., Petigura, E. A., et al. 2014, Proceedings of the National Academy of Science, 111, 12655

Masset, F. \& Snellgrove, M. 2001, MNRAS, 320, L55 
Masset, F. S. \& Casoli, J. 2010, ApJ, 723, 1393

Mayor, M., Marmier, M., Lovis, C., et al. 2011, arXiv, astro-ph 1109.2497

Mayor, M. \& Queloz, D. 1995, Nature, 378, 355

Mizuno, H. 1980, Progress of Theoretical Physics, 64, 544

Mollière, P. \& Mordasini, C. 2012, A\&A, 547, A105

Mordasini, C. 2014, A\&A, 572, A118

Mordasini, C., Alibert, Y., \& Benz, W. 2006, in Tenth Anniversary of 51 Peg-b: Status of and prospects for hot Jupiter studies, ed. L. Arnold, F. Bouchy, \& C. Moutou (Paris: Frontier Group), 84-86

Mordasini, C., Alibert, Y., \& Benz, W. 2009a, A\&A, 501, 1139

Mordasini, C., Alibert, Y., Benz, W., Klahr, H., \& Henning, T. 2012a, A\&A, 541, 97

Mordasini, C., Alibert, Y., Benz, W., \& Naef, D. 2009b, A\&A, 501, 1161

Mordasini, C., Alibert, Y., Georgy, C., et al. 2012b, A\&A, 547, 112

Mordasini, C., Alibert, Y., Klahr, H., \& Henning, T. 2012c, A\&A, 547, 111

Mordasini, C., Klahr, H., Alibert, Y., Miller, N., \& Henning, T. 2014, A\&A, 566, A141

Mordasini, C., Marleau, G.-D., \& Mollière, P. 2017, A\&A, 608, A72

Mordasini, C., Mollière, P., Dittkrist, K.-M., Jin, S., \& Alibert, Y. 2015, International Journal of Astrobiology, 14, 201

Mordasini, C., van Boekel, R., Mollière, P., Henning, T., \& Benneke, B. 2016, ApJ, 832, 41

Mortier, A., Santos, N. C., Sousa, S., et al. 2013, A\&A, 551, A112

Moutou, C., Deleuil, M., Guillot, T., et al. 2013, Icarus, 226, 1625

Movshovitz, N., Bodenheimer, P. H., Podolak, M., \& Lissauer, J. J. 2010, Icarus, 209, 616

Mulders, G. D., Pascucci, I., \& Apai, D. 2015, ApJ, 814, 130

Müller, S., Helled, R., \& Mayer, L. 2018, ArXiv e-prints

Nakazawa, K., Ida, S., \& Nakagawa, Y. 1989, A\&A, 220, 293

Nayakshin, S. 2010, MNRAS, 408, L36

Nayakshin, S. \& Fletcher, M. 2015, MNRAS, 452, 1654

Ndugu, N., Bitsch, B., \& Jurua, E. 2018, MNRAS, 474, 886

Ogihara, M., Morbidelli, A., \& Guillot, T. 2015, A\&A, 584, L1

Ormel, C. W. 2014, ApJ, 789, L18

Ormel, C. W. 2017, in Astrophysics and Space Science Library, Vol. 445, Astrophysics and Space Science Library, ed. M. Pessah \& O. Gressel, 197

Ormel, C. W., Dullemond, C. P., \& Spaans, M. 2010, Icarus, 210, 507

Ormel, C. W., Ida, S., \& Tanaka, H. 2012, ApJ, 758, 80

Ormel, C. W. \& Klahr, H. H. 2010, A\&A, 520, A43

Ormel, C. W., Shi, J.-M., \& Kuiper, R. 2015, MNRAS, 447, 3512

Owen, J. E. \& Wu, Y. 2013, ApJ, 775, 105

Owen, J. E. \& Wu, Y. 2017, ApJ, 847, 29

Paardekooper, S.-J. 2014, MNRAS, 444, 2031

Paardekooper, S.-J., Baruteau, C., Crida, A., \& Kley, W. 2010, MNRAS, 401, 1950

Panić, O., Hogerheijde, M. R., Wilner, D., \& Qi, C. 2009, A\&A, 501, 269

Perri, F. \& Cameron, A. G. W. 1974, Icarus, 22, 416

Petigura, E. A., Howard, A. W., \& Marcy, G. W. 2013, Proceedings of the National Academy of Science, 110, 19273

Petigura, E. A., Marcy, G. W., Winn, J. N., et al. 2018, AJ, 155, 89

Pierens, A. 2015, MNRAS, 454, 2003

Pinilla, P., Birnstiel, T., \& Walsh, C. 2015, A\&A, 580, A105

Piso, A.-M. A., Öberg, K. I., Birnstiel, T., \& Murray-Clay, R. A. 2015, ApJ, 815, 109

Piso, A.-M. A. \& Youdin, A. N. 2014, ApJ, 786, 21

Podolak, M., Pollack, J. B., \& Reynolds, R. T. 1988, Icarus, 73, 163

Pollack, J. B., Hubickyj, O., Bodenheimer, P. H., et al. 1996, Icarus, 124, 62

Reffert, S., Bergmann, C., Quirrenbach, A., Trifonov, T., \& Künstler, A. 2015, A\&A, 574, A116

Safronov, V. S. 1969, Evolution of the Protoplanetary Cloud and Formation of the Earth and the Planets (Nauka, Moscow) 
Sahlmann, J., Segransan, D., Queloz, D., et al. 2011, A\&A, 525, 95

Sallum, S., Follette, K. B., Eisner, J. A., et al. 2015, Nature, 527, 342

Salpeter, E. E. 1955, ApJ, 121, 161

Sándor, Z., Lyra, W., \& Dullemond, C. P. 2011, ApJ, 728, L9

Santos, N. C., Adibekyan, V., Figueira, P., et al. 2017, A\&A, 603, A30

Santos, N. C., Israelian, G., \& Mayor, M. 2004, A\&A, 415, 1153

Santos, N. C., Israelian, G., Mayor, M., et al. 2005, A\&A, 437, 1127

Schneider, J., Dedieu, C., Le Sidaner, P., Savalle, R., \& Zolotukhin, I. 2011, A\&A, 532, A79

Shakura, N. I. \& Sunyaev, R. A. 1973, A\&A, 24, 337

Shu, F. H. 1977, ApJ, 214, 488

Shu, F. H., Tremaine, S., Adams, F. C., \& Ruden, S. P. 1990, ApJ, 358, 495

Tanaka, H., Takeuchi, T., \& Ward, W. R. 2002, ApJ, 565, 1257

Thommes, E. W., Duncan, M. J., \& Levison, H. F. 2003, Icarus, 161, 431

Thommes, E. W., Matsumura, S., \& Rasio, F. A. 2008, Science, 321, 814

Udry, S., Mayor, M., \& Santos, N. C. 2003, A\&A, 407, 369

Udry, S. \& Santos, N. C. 2007, Annual Review of A\&A, 45, 397

Van Eylen, V., Agentoft, C., Lundkvist, M. S., et al. 2017, ArXiv e-prints

Veras, D. \& Armitage, P. J. 2004, MNRAS, 347, 613

Visser, R. G. \& Ormel, C. W. 2016, A\&A, 586, A66

Walsh, K. J., Morbidelli, A., Raymond, S. N., O’Brien, D. P., \& Mandell, A. M. 2011, Nature, 475, 206

Ward, W. R. 1986, Icarus, 67, 164

Weidenschilling, S. 1977, Astrophysics and Space Science, 51, 153

Winn, J. N. \& Fabrycky, D. C. 2015, ARA\&A, 53, 409

Wolfgang, A., Rogers, L. A., \& Ford, E. B. 2016, ApJ, 825, 19

Wright, J. T., Fakhouri, O., Marcy, G. W., et al. 2011, PASP, 123, 412

Yu, L., Donati, J.-F., Hébrard, E. M., et al. 2017, MNRAS, 467, 1342 